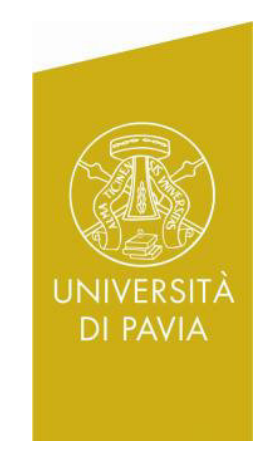

Department of Economics and Management DEM Working Paper Series

\title{
A Two-Stage Estimator for Heterogeneous Panel Models with Common Factors
}

Carolina Castagnetti

(Università di Pavia)

Eduardo Rossi

(Università di Pavia)

Lorenzo Trapani

(City University, London)

\# 66 (01-14)

Via San Felice, 5

I-27100 Pavia

http://epmq.unipv.eu/site/home.html

Janauary 2014 


\title{
A Two-Stage Estimator for Heterogeneous Panel Models with
}

\section{Common Factors*}

\author{
Carolina Castagnetti ${ }^{1}$, Eduardo Rossi ${ }^{\dagger 1}$, and Lorenzo Trapani ${ }^{2}$ \\ ${ }^{1}$ Dipartimento di Scienze Economiche e Aziendali, Università di Pavia, Italy \\ ${ }^{2}$ Faculty of Finance, Cass Business School, City University, London (UK)
}

January 31, 2014

\begin{abstract}
This paper considers estimation in a stationary heterogeneous panel model where common unknown factors are present. A two-stage estimator is proposed. This estimator is based on the CCE estimator (Pesaran, 2006) in the first stage and on a similar approach to the Interactive Effect estimator (Bai, 2009) in the second stage. The asymptotic properties of this estimator are provided alongside of the comparative finite-sample properties of a range of estimators by means of Monte Carlo experiments.
\end{abstract}

Keywords: Large panels; Factor error structure; Principal components; Common regressors; Cross-section dependence.

JEL - Classification: C33, C38

${ }^{*}$ We wish to thank the participants to the 2013 PRIN MISURA workshop (University of Venice, September 2013); to the 19th International Panel Data Conference (Cass Business School, London, July 2013). Special thanks to Luca Fanelli for providing us with valuable comments. Carolina Castagnetti and Eduardo Rossi acknowledge financial support from PRIN MISURA. The usual disclaimer applies.

${ }^{\dagger}$ Correspondence to: Dipartimento di Scienze Economiche e Aziendali, Via San Felice 5, 27100 Pavia. E-mail: erossi@eco.unipv.it 


\section{Introduction}

Consider the model

$$
y_{i t}=\beta_{i}^{\prime} x_{i t}+\gamma_{i}^{\prime} f_{t}+\epsilon_{i t} \quad i=1, \ldots, n \quad t=1, \ldots, T,
$$

where $i=1, \ldots, n$ and $t=1, \ldots, T ; x_{i t}$ is a $k \times 1$ vector of individual-specific components, and $f_{t}$ is the $r \times 1$ vector of unobserved factors. We further allow the $x_{i t}$ s to be cross sectionally correlated by being linearly dependent on a set of common unobserved factor

$$
x_{i t}=A_{i} f_{t}+u_{i t}
$$

$A_{i}$ is $k \times r$ is a factor loading matrix with nonrandom components and $u_{i t}$ are the specific components of $x_{i t}$ distributed independently of the common effects and across $i$. The main advantage of model (1)-(2) is its generality: the model considers the presence of cross sectional correlation in the $y_{i t} \mathrm{~s}$; it does not rule out the possibility that the idiosyncratic regressors $x_{i t}$ are also strongly cross correlated; and it also allows for nontrivial correlation between the observable regressors $x_{i t}$ and the unobserved ones $f_{t}$. By virtue of its generality, model (1)-(2) nests, as pointed out in Pesaran (2006), several popular specifications.

On account of the huge application potential of (1)-(2), the literature on panel data has focused on developing the inferential theory for the slope coefficients $\beta_{i}$ in presence of cross sectional dependence arising from a common factor structure. Several contributions have pointed out that slope estimation may be inconsistent when such common factors are correlated with the other regressors - see e.g. Pesaran (2006). Pesaran (2006) suggests a new approach, based on running a regression augmented with the cross sectional averages of dependent and independent variables, known as the Common Correlated Coefficients (CCE) estimator. The set up in Pesaran (2006) is very general and his results can also be extended to the case of non stationary common factors (Kapetanios, Pesaran, and Yamagata, 2011) and also hold in presence of spatial correlation in the error term (Pesaran and Tosetti, 2011). In another seminal contribution, Bai (2009) proposes an alternative estimator, called Interactive Fixed Effects (IFE), which combines standard OLS with the Principal Components estimation of the unobservable common factors. Whilst the contribution by Bai (2009) assumes homogeneous slopes, a recent contribution by Song (2013) extends the IFE estimator to the case of heterogeneous panels with cross-section dependence, extending the 
estimation theory to the case of dynamic panels. A common feature to the contributions by Pesaran (2006), Bai (2009) and Song (2013) is that they deal mainly with the estimation of the slope parameters, and inference on the unobserved common factors and their loadings is not fully developed.

There are, however, examples where the parameters of interest are not only the slope coefficients but also the common factors $f_{t}$ and their loadings $\lambda_{i}$. For example, several contributions have studied the possibility of augmenting a standard regression model with the estimates of common factors from a large set of data (see, for example, Kapetanios and Pesaran, 2007, Ludvigson and $\mathrm{Ng}, 2007$ and Ludvigson and Ng, 2009). Estimated factors may also be used e.g. as inputs for diffusion index forecasting (see Stock and Watson, 2005) and as regressors in factor augmented VARs (see Bernanke, Boivin, and Eliasz, 2005). Castagnetti, Rossi, and Trapani (2012) develop an inferential theory for the unobserved common factors and loadings.

In this paper, we build on (1)-(2), and extend the existing inferential theory on the average slope - say $\beta=E\left(\beta_{i}\right)$, in a random coefficient framework. Specifically, we propose a two-stage estimator of the average slope coefficient, based on the CCE estimator in the first stage. Such estimator can be thought of as the IFE estimator with only one iteration, and thus it complements the results in Bai (2009) and Song (2013) by proposing a simpler version of the estimators defined therein. Heuristically, the two-stage approach should combine the efficiency that can be naturally expected from an iterative procedure such as the IFE, with a greater computational simplicity and no risk of the iterative procedure to fail to achieve convergence. The small sample properties of the proposed estimator, analysed through synthetic data, show that the proposed estimator has good finite sample properties. The Monte Carlo experiments show that the two-stage estimator is, in finite samples, less biased than IFE and more efficient. The two-stage procedure, which represents a simple alternative to the IFE, offers the best trade-off between efficiency and computational reliability.

The remainder of the paper is organized as follows. Section 2 discusses the model and the main assumptions. Section 2illustrates the two-stage estimators, and reports the asymptotic results. Section 3 reports the details of the Monte Carlo experiments as well as the main results from the simulations. Section 4 concludes. Preliminary Lemmas are in Appendix A, and the proof of the main result is in Appendix B.

NOTATION. We use " $\longrightarrow$ " to denote the ordinary limit; " $\stackrel{d}{\longrightarrow}$ " and " $\stackrel{p}{\longrightarrow}$ " to denote convergence in distribution and in probability respectively, and "a.s." for "almost surely". The Frobenius norm 
of a matrix $A$ is denoted as $\|A\|=\sqrt{\operatorname{tr}\left(A^{\prime} A\right)}$, where $\operatorname{tr}(A)$ denotes the trace of $A$. Finite constants that do not depend on the sample size are denoted as $M, M^{\prime}, M^{\prime \prime}$, etc... Other notation is defined throughout the paper and in Appendix.

\section{The two stage estimator}

In this section, we define the two-stage estimator of the average slope, and we derive its asymptotics properties.

Recall model (1)-(2):

$$
\begin{aligned}
y_{i t} & =\beta_{i}^{\prime} x_{i t}+\gamma_{i}^{\prime} f_{t}+\epsilon_{i t}, \\
x_{i t} & =A_{i} f_{t}+u_{i t},
\end{aligned}
$$

and its matrix form:

$$
\begin{aligned}
y_{i} & =X_{i} \beta_{i}+F \gamma_{i}+\epsilon_{i}, \\
X_{i} & =F A_{i}+u_{i},
\end{aligned}
$$

with $y_{i}=\left[y_{i 1}, \ldots, y_{i T}\right]^{\prime}, X_{i}=\left[x_{i 1}|\ldots| x_{i T}\right]^{\prime}, F=\left[f_{1}|\ldots| f_{T}\right]^{\prime}$ and $\epsilon_{i}=\left[\epsilon_{i 1}, \ldots, \epsilon_{i T}\right]^{\prime}$. In order to estimate the individual slopes $\beta_{i}$, the CCE estimator proposed in Pesaran (2006) is given by augmenting the OLS regression of $y_{i t}$ on $x_{i t}$ with the cross-section averages $\bar{z}_{t}=\frac{1}{n} \sum_{i=1}^{n} z_{i t}$, with $z_{i t}=\left[y_{i t}, x_{i t}^{\prime}\right]^{\prime}$. Based on this intuition, the CCE estimator is defined as

$$
\tilde{\beta}_{i}=\left(X_{i}^{\prime} \bar{M} X_{i}\right)^{-1} X_{i}^{\prime} \bar{M} y_{i}
$$

with $\bar{M}=I_{T}-\bar{Z}\left(\bar{Z}^{\prime} \bar{Z}\right)^{-1} \bar{Z}^{\prime}$, and $\bar{Z}$ is the $T \times(k+1)$ matrix of observations on $\bar{z}_{t}$. Pesaran (2006) proposes two estimators for the means of the individual specific slope coefficients: The Common Correlated Effects Mean Group (CCEMG) estimator, a generalization of the estimator proposed by Pesaran and Smith (1995), and a generalization of the fixed effects estimator, the Common Correlated Effects Pooled (CCEP) estimator. The CCEMG estimator is a simple average of the individual CCE estimators, $\tilde{\beta}_{i}$ of $\beta_{i}$ :

$$
\tilde{\beta}_{C C E M G}=\frac{1}{n} \sum_{i=1}^{n} \tilde{\beta}_{i}
$$


Assuming $\beta_{i}=\beta$, the CCEP estimator, which allows for the possibility of cross-section dependence, in its simplest form is given by:

$$
\tilde{\beta}_{C C E P}=\left(\sum_{i=1}^{n} X_{i}^{\prime} \bar{M} X_{i}\right)^{-1} \sum_{i=1}^{n} X_{i}^{\prime} \bar{M} y_{i}
$$

The two-stage estimation procedure of the average slope $\beta=E\left(\beta_{i}\right)$ is based on the following two steps:

Step 1 Obtain an estimate of the common factors $f_{t}$ in (1)-(2):

1.(a) estimate the $\beta_{i}$ s using the CCE estimator defined in (3), and compute the residuals $\tilde{v}_{i}=y_{i}-X_{i} \tilde{\beta}_{i}$

1.(b) Apply the Principal Component estimator (henceforth, PC) to $\tilde{v}_{i}$, obtaining $\hat{F}$ with the restriction $\hat{F}^{\prime} \hat{F}=T I_{r}$.

Step 2 Re-estimate the slopes $\beta_{i}$ and compute their average:

2.(a) Apply OLS to

$$
y_{i t}=\beta_{i}^{\prime} x_{i t}+\gamma_{i}^{\prime} \hat{f}_{t}+\epsilon_{i t},
$$

obtaining

$$
\hat{\beta}_{i}=\left(X_{i}^{\prime} \hat{M}_{F} X_{i}\right)^{-1}\left(X_{i}^{\prime} \hat{M}_{F} y_{i}\right)
$$

with $\hat{M}_{F}=I_{T}-\hat{F} \hat{F}^{\prime} / T$

2.(b) define the Augmented Mean Group (AMG hereafter) estimator of $\beta$

$$
\hat{\beta}^{A M G}=\frac{1}{n} \sum_{i=1}^{n} \hat{\beta}_{i} .
$$

In Step 1.(b), it is worth noting that the number of unobserved factors, $r$, can be assumed to be fixed but it is unknown, and it needs to be estimated as well. Indeed, given a consistent estimator of the $\beta_{i}$ s such as e.g. the CCE estimator, the residuals $\tilde{v}_{i}$ have a pure factor model structure, viz. $\tilde{v}_{i}=\gamma_{i}{ }^{\prime} f_{t}+\epsilon_{i t}+\left(\beta_{i}-\tilde{\beta}_{i}\right)^{\prime} x_{i t}$. Bai (2009) and Pesaran (2006, p.30) show that the error component $\left(\beta_{i}-\tilde{\beta}_{i}\right)^{\prime} x_{i t}$ does not affect the determination of the number of common factors, which can be 
therefore estimated using e.g. the information criteria developed by Bai and Ng (2002). Similarly, it is well-known that common factors and loadings are not separately identified, and therefore can be estimated only up to a rotation. As far as our setup is concerned, knowing a rotation of the common factors and loadings is as good as knowing the true factors and loadings.

Turning to Step 2, note that in Step 2.(a), on account of (8), we have

$$
\hat{\beta}_{i}-\beta_{i}=\left(\frac{X_{i}^{\prime} \hat{M}_{F} X_{i}}{T}\right)^{-1}\left[\frac{X_{i}^{\prime} \hat{M}_{F} F}{T} \gamma_{i}+\frac{X_{i}^{\prime} \hat{M}_{F} \epsilon_{i}}{T}\right]
$$

this can be compared with the IFE estimator in Song (2013). As a consequence, in Step 2.(b) the AMG estimator can be expressed as

$$
\hat{\beta}^{A M G}-\beta=\frac{1}{n} \sum_{i=1}^{n} \nu_{i}+\frac{1}{n} \sum_{i=1}^{n}\left(\frac{X_{i}^{\prime} \hat{M}_{F} X_{i}}{T}\right)^{-1}\left[\frac{X_{i}^{\prime} \hat{M}_{F} F}{T} \gamma_{i}+\frac{X_{i}^{\prime} \hat{M}_{F} \epsilon_{i}}{T}\right] .
$$

Finally, in addition to $\hat{\beta}^{A M G}$ we define the Augmented Pooled (AP) estimator

$$
\hat{\beta}^{A P}=\left(\frac{1}{n} \sum_{i=1}^{n} X_{i}^{\prime} \hat{M}_{F} X_{i}\right)^{-1}\left(\frac{1}{n} \sum_{i=1}^{n} X_{i}^{\prime} \hat{M}_{F} y_{i}\right)
$$

In order to study the asymptotic properties of $\hat{\beta}^{A M G}$ and of $\hat{\beta}^{A P}$, consider the following assumptions.

Assumption 1. [error terms: serial and cross sectional dependence] (i) $E\left(\epsilon_{i t}\right)=0$ and $E\left|\epsilon_{i t}\right|^{12}<\infty$; (ii) (a) $\sum_{t=1}^{T}\left|E\left(\epsilon_{i t} \epsilon_{i s}\right)\right| \leq M$ for all $i$ and $s$, (b) $\sum_{i=1}^{n} \sum_{j=1}^{n}\left|E\left(\epsilon_{i t} \epsilon_{j s}\right)\right| \leq M n$ for all $t$ and $s,(\mathrm{c}) \sum_{t=1}^{T} \sum_{s=1}^{T}\left|E\left(\epsilon_{i t} \epsilon_{i s}\right)\right| \leq M T$ for all $i,(\mathrm{~d}) \sum_{i=1}^{n} \sum_{j=1}^{n} \sum_{t=1}^{T} \sum_{s=1}^{T}\left|E\left(\epsilon_{i t} \epsilon_{j s}\right)\right| \leq$ $M(n T)$; (iii) (a) $E\left|(n T)^{-1 / 2} \sum_{i=1}^{n} \sum_{t=1}^{T} \epsilon_{i t}\right|^{2} \leq M$, (b) $\sum_{t=1}^{T} \sum_{s=1}^{T} \sum_{v=1}^{T} \sum_{u=1}^{T}\left|E\left(\epsilon_{i t} \epsilon_{i s} \epsilon_{i u} \epsilon_{i v}\right)\right|$ $\leq M T^{2}$, (c) $\sum_{i=1}^{n} \sum_{j=1}^{n} \sum_{t=1}^{T} \sum_{u=1}^{T}\left|E\left(\epsilon_{i t} \epsilon_{i s} \epsilon_{j u} \epsilon_{j s}\right)\right| \leq M(n T)$ for all $u$, (d) $\sum_{i=1}^{n} \sum_{j=1}^{n} \sum_{t=1}^{T}$ $\sum_{s=1}^{T}\left|E\left(\epsilon_{i t} \epsilon_{k t} \epsilon_{j s} \epsilon_{k s}\right)\right| \leq M(n T)$ for all $k$; (iv) (a) $E\left|\sum_{t=1}^{T} \epsilon_{i t}\right|^{r} \leq M E\left|\sum_{t=1}^{T} \epsilon_{i t}^{2}\right|^{r / 2}$ for all $i, r<12$, (b) $E\left|\sum_{i=1}^{n} \epsilon_{i t}\right|^{r} \leq M E\left|\sum_{i=1}^{n} \epsilon_{i t}^{2}\right|^{r / 2}$ for all $t, r<12$.

Assumption 2. [regressors and common factors] (i) $E\left\|\epsilon_{i t}^{x}\right\|^{12}<\infty$ and $E\left\|f_{t}\right\|^{12}<\infty$; (ii) $T^{-1} \sum_{t=1}^{T} f_{t} f_{t}^{\prime} \stackrel{p}{\rightarrow} \Sigma_{f}$ as $T \rightarrow \infty$ with $\Sigma_{f}$ non-singular; (iii) $\left\{\epsilon_{i t}^{x}, f_{t}\right\}$ and $\left\{\epsilon_{j s}\right\}$ are mutually independent for all $i, j, t, s$; (iv) $E\left|\sum_{t=1}^{T} x_{i t} \epsilon_{i t}\right|^{r} \leq M E\left|\sum_{t=1}^{T}\left(x_{i t} \epsilon_{i t}\right)^{2}\right|^{r / 2}$ for all $i, r \leq 6$.

Assumption 3. [slopes and loadings] (i) $\beta_{i}=\beta+v_{i}$ with $\|\beta\| \leq M$ and $v_{i}$ is i.i.d. across $i$ with mean zero and independent of $\left\{\epsilon_{j t}, \epsilon_{j t}^{x}, f_{t}\right\}$ for all $i, j$, $t$; (ii) $E\left\|v_{i}\right\|^{2+\delta}<\infty$ for some $\delta>0$; (iii) the $\gamma_{i}$ s are non stochastic and such that $\max _{i}\left\|\gamma_{i}\right\|<\infty$ and $n^{-1} \sum_{i=1}^{n} \gamma_{i} \gamma_{i}^{\prime} \rightarrow \Sigma_{\gamma}$ as $n \rightarrow \infty$ 
with $\Sigma_{\gamma}$ non-singular.

Assumption 4. [Step 1 estimation] (i) $l_{\min }\left(\frac{X_{i}^{\prime} \bar{M}_{w} X_{i}}{T}\right)>0 ; l_{\min }\left(\frac{X_{i}^{\prime} M_{F} X_{i}}{T}\right)>0$ and $l_{\min }\left(\frac{F^{\prime} M_{X i} F}{T}\right)>$ 0 a.s. for all $i$, where $l_{\min }(\cdot)$ denotes the smallest eigenvalue; (ii) $C \equiv n^{-1} \sum_{i=1}^{n} C_{i}$ has rank $r \leq m+1$.

Assumptions 1-4 are the same set of assumptions as in Castagnetti, Rossi, and Trapani (2012); basically, they are needed in order to prove the consistency of the estimated common factors and loadings. Assumption 4 is specific to the CCE estimator, employed in Step 1. In Assumption 1, serial and cross sectional dependence are allowed for the error term $\epsilon_{i t}$. The rest of the assumption is similar to those in Bai (2009) and Pesaran (2006), and hold immediately if $\epsilon_{i t}$ is assumed to be independent. Note that existence of the 12-th moment of $\epsilon_{i t}$ is required - this is stronger than what the literature normally considers, and in our context it is needed in order to derive consistency of $\hat{\gamma}_{i}$ and $\hat{f}_{t}$. Finally, part (iv) contains Burkholder-type inequalities: these could be shown directly under more specific assumptions on the degree of serial and cross sectional dependence. For example, part (a) holds immediately if one assumes that $\epsilon_{i t}$ is a Martingale Difference Sequence (MDS) across $t$ (the same holds for part (b), under the MDS assumption across $i$ ) - see e.g. Lin and Bai (2010, p.108). As far as Assumption 2 is concerned, we allow for serial and cross sectional dependence in both the $\epsilon_{i t}^{x}$ and in the common factors $f_{t}$. The requirement in part (ii) is standard in the literature (see e.g. Assumption B in Bai, 2009), and it entails that common factors are "strong" in the sense of Chudik, Pesaran, and Tosetti (2011) (see in particular Assumption 3). Finally, according to part (iii), the $x_{i t}$ s are strictly exogenous. Assumption 3 is standard. Assumption 4 is specific to the CCE estimator of the $\beta_{i}$ s, employed in Step 1. Particularly, the rank condition in part (ii) is the same as equation (21) in Pesaran (2006), and it guarantees the consistency of the $\tilde{\beta}_{i} \mathrm{~s}$.

We are now ready to present the asymptotics (rates of convergence and limiting distribution) for the two-stage estimators $\hat{\beta}^{A M G}$ and $\hat{\beta}^{A P}$. Let $E\left(v_{i} v_{i}^{\prime}\right)=\Omega_{v}$, and consider the Augmented Mean Group estimator defined in (8).

Theorem 1 Let Assumptions 1-4 hold with $\Omega_{v} \neq 0$. Then, as $(n, T) \rightarrow \infty$

$$
\hat{\beta}^{A M G}-\beta=O_{p}\left(\frac{1}{\sqrt{n}}\right)+O_{p}\left(\frac{1}{\delta_{n T}^{2}}\right)
$$


similar results hold for $\hat{\beta}^{A P}-\beta$. Under $\frac{\sqrt{n}}{T} \rightarrow 0$, it holds that

$$
\begin{aligned}
& \sqrt{n}\left(\hat{\beta}^{A M G}-\beta\right) \stackrel{d}{\rightarrow} N\left(0, \Omega_{v}\right), \\
& \sqrt{n}\left(\hat{\beta}^{A P}-\beta\right) \stackrel{d}{\rightarrow} N\left(0, \tilde{\Omega}_{v}\right),
\end{aligned}
$$

where

$$
\tilde{\Omega}_{v}=\lim _{n, T \rightarrow \infty}\left(\frac{1}{n} \sum_{i=1}^{n} X_{i}^{\prime} M_{F} X_{i}\right)^{-1}\left[\frac{1}{n} \sum_{i=1}^{n} X_{i}^{\prime} M_{F} X_{i} \Omega_{v} X_{i}^{\prime} M_{F} X_{i}\right]\left(\frac{1}{n} \sum_{i=1}^{n} X_{i}^{\prime} M_{F} X_{i}\right)^{-1}
$$

If Assumptions 1-4 hold and $\Omega_{v}=0$, then as $(n, T) \rightarrow \infty$ we have $\hat{\beta}^{A M G}-\beta=O_{p}\left(\delta_{n T}^{-2}\right)$. The same rates holds for $\hat{\beta}^{A P}$.

Theorem 1 reports rates of convergence and asymptotic distributions of both the AMG estimator $\hat{\beta}^{A M G}$ and the AP one $\hat{\beta}^{A P}$; further, rates are studied under slope homogeneity. From a theoretical point of view, the theorem states that, in essence, there is no improvement with respect to the first stage estimator (see Pesaran, 2006). Indeed, the limiting distribution is driven by the term $n^{-1 / 2} \sum_{i=1}^{n}\left(\beta_{i}-\beta\right)$, which the iterative procedure does not improve. Indeed, as far as rates are concerned, equation (11) also contains the term $O_{p}\left(\frac{1}{T}\right)$, which arises from having the generated regressors $\hat{f}_{t}$ in the second stage equation (6). Similarly, under slope homogeneity, both $\hat{\beta}^{A M G}$ and $\hat{\beta}^{A P}$ are consistent at a rate $O_{p}\left(\delta_{n T}^{-2}\right)$, slower than the Mean Group and the Pooled estimators defined from $\tilde{\beta}_{i}$, which are consistent at a rate $O_{p}\left(\frac{1}{\sqrt{n T}}\right)$.

The covariance matrix $\Omega_{v}$ can be estimated using $\hat{\Omega}_{v}=n^{-1} \sum_{i=1}^{n}\left(\hat{\beta}_{i}-\hat{\beta}^{A M G}\right)\left(\hat{\beta}_{i}-\hat{\beta}^{A M G}\right)^{\prime}$; the same passages as in Pesaran (2006) would entail $\hat{\Omega}_{v} \stackrel{p}{\rightarrow} \Omega_{v}$ as $(n, T) \rightarrow \infty$. By (14), $\tilde{\Omega}_{v}$ can also be readily estimated using $\hat{\Omega}_{v}$ as a plug-in estimator.

\section{Monte Carlo Experiments}

In this section, we compare the finite sample properties of several estimators (mainly based on the CCE of Pesaran, 2006, and the IFE of Bai, 2009, and Song, 2013) with the two-stage estimator proposed in Section 2, through synthetic data.

Simulations are carried out under different DGPs; specifically, we consider (1) a homogeneous panel with observed and unobserved common factors; (2) a heterogeneous panel with observed and unobserved common factors; and (3) a heterogeneous panel with unobserved common factors 
only. We refer to the subsections hereafter for a more through discussion of each specification. In each subsection, we consider combinations of $n$ and $T$ based on $n, T \in\{10,30,50,100,200\}$. Each experiment involves 5,000 replications.

It is worth noting that the estimators considered in this section require the determination of the number of the common factors $r$. This would be customarily done by using some information criteria such as the ones in Bai and Ng (2002). However, such method could, in small samples, produce an inconsistent estimate of $r$, thereby leading to incorrect inference. We refer to the simulation results in Coakley, Fuertes, and Smith (2002) and Kapetanios and Pesaran (2007). Thus, we firstly assume that the number of factors is known throughout the simulations, to examine the small sample properties of the estimators without them being affected by the issue of estimating $r$. We then introduce the number of factors estimation problem in order to take into account its impact on the estimation errors.

We consider the following DGP:

$$
\begin{gathered}
y_{i t}=\alpha_{1} d_{1 t}+\alpha_{2} d_{2 t}+\beta_{1} x_{1 i t}+\beta_{2} x_{2 i t}+\gamma_{i} f_{t}+\epsilon_{i t}, \\
x_{1 i t}=a_{1}+\gamma_{i} f_{t}+\gamma_{i}+f_{t}+v_{1 i t}, \\
x_{2 i t}=a_{2}+\gamma_{i} f_{t}+\gamma_{i}+f_{t}+v_{2 i t} .
\end{gathered}
$$

In (15)-(17), we consider: two individual specific components, $x_{1 i t}$ and $x_{2 i t}$; two observed common factors, $d_{1 t}$ and $d_{2 t}$; and one unobserved common factor $f_{t}$. The error term $\epsilon_{i t}$ is generated as i.i.d. $N(0,2)$; further we set $a_{1}=a_{2}=1$. The observed factors are generated as

$$
\begin{aligned}
& d_{1 t}=1, \\
& d_{2 t}=f_{t}+v_{t}^{d},
\end{aligned}
$$

where $v_{t}^{d}$ is generated as i.i.d. $N(0,1)$ and independent of all other regressors. The homogeneous coefficients are set to $\beta=\left(\beta_{1}, \beta_{2}\right)=(1,3)$ and $\alpha=\left(\alpha_{1}, \alpha_{2}\right)=(5,4)$. The variables $\gamma_{i}, f_{t}, v_{j i t}$ are all generated as i.i.d. $N(0,1)$. This DGP is identical to Bai (2009) DGP for the case of common regressors.

Following Bai (2003), we compute for each iteration the correlation coefficient between $\left\{\hat{f}_{A P, t}\right\}_{t=1}^{T}$ and $\left\{f_{t}\right\}_{t=1}^{T}$, where $\hat{f}_{A P, t}$ is the estimated factor obtained from the residuals $\tilde{v}_{i t}=y_{i t}-x_{i t}^{\prime} \tilde{\beta}_{C C E P}$. We compare them with those obtained using the IFE estimate for the factor, $\hat{f}_{I F E, t}$. Table 1 below 
reports the average correlation coefficients for both estimation methods

\section{[Insert Table 1 somewhere here]}

The results in Table 1 suggest that both factor estimates are highly correlated with the unobserved factor. The last two columns of the table report the average number of iterations and the number of failures for each Monte Carlo simulation, respectively. Failure to achieve convergence for iterative procedures seems to occur particularly for small samples, which is particularly evident for $n=30, T=10$.

The bias and the RMSE of the estimates are in Tables 2 and 3 respectively. Further, bias and RMSE are also computed for the infeasible pooled estimator, calculated as:

$$
\widehat{\beta}_{\text {inf,pooled }}=\left[\sum_{i=1}^{n}\left(X_{i}{ }^{\prime} M_{F} X_{i}\right)\right]^{-1} \sum_{i=1}^{n}\left(X_{i}{ }^{\prime} M_{F} y_{i}\right) .
$$

In principle, the infeasible estimator constitutes a lower bound to the bias and efficiency.

\section{[Insert Tables 2 and 3 somewhere here]}

We observe that for $n \leq 30$, the AP estimator slightly outperforms both the CCEP and the IFE estimator in terms of bias and RMSE. The situation is reversed when $n$ is larger than 30. In this case, CCEP and IFE are less biased than AP. In general, the AP estimator and the IFE are quite close in terms of bias and RMSE. For all estimators considered, the RMSE decreases when $n$ increases, for each value of $T$. As expected, the RMSE gets smaller as long as both $n$ and $T$ increase.

\subsection{Heterogeneous panel with observed and unobserved common factors}

The setup in (15)-(17) is extended to the case of heterogenous slopes, viz.

$$
\alpha_{1 i}=5+\eta_{i 3}, \quad \alpha_{2 i}=4+\eta_{i 4},
$$

and

$$
\beta_{1 i}=1+\eta_{i 1}, \quad \beta_{2 i}=3+\eta_{i 2},
$$


where $\eta_{i j} \sim$ i.i.d.N $(0,0.04)$ for $j=1, \ldots, 4$. For each experiment we compute the CCEMG by Pesaran (2006), the Augmented Mean Group (AMG) and the Song (2013) estimator as well as the infeasible MG estimator, assuming $f_{t}$ is observable. Namely the infeasible MG estimator is computed as:

$$
\widehat{\beta}_{i n f, M G}=\frac{1}{n} \sum_{i=1}^{n}\left(X_{i}{ }^{\prime} M_{F} X_{i}\right)^{-1}\left(X_{i}{ }^{\prime} M_{F} y_{i}\right) .
$$

The Song (2013) estimator is computed by allowing up to 200 iterations for each simulation; as a convergence criterion, we employ $\|\operatorname{vec}(\hat{\beta})-\operatorname{vec}(\beta)\|$, where $\operatorname{vec}(\beta)=\left(\beta_{1}^{\prime}, \ldots, \beta_{n}^{\prime}\right)^{\prime}$ and $\beta_{i}=$ $\left(\beta_{1 i}, \beta_{2 i}\right)^{\prime}$, and we set the tolerance coefficient equal to 0.0001 .

Bias and RMSE of the various estimators are in Tables 4 and 5 respectively.

\section{[Insert Tables 4 and 5 somewhere here]}

The tables show that, when slope heterogeneity is considered, results are radically different from the homogeneous case in the previous subsection. In terms of bias, the AMG dominates both the CCEMG and the Mean Group estimator based on the IFE individual estimates studied in Song (2013). Similar conclusions can be drawn when considering the RMSEs in Table 5, although the differences between CCEMG and AMG are very small.

Finally, we consider the average correlation between estimated factors and true ones, reported in Table 6.

\section{[Insert Table 6 somewhere here]}

Interestingly, Table 6 shows that the two-step procedure yields estimated factors that are more correlated with the true ones than other procedures. Again, iterative procedures are computationally costly, even more than in the case of a homogeneous panel.

\subsection{Heterogeneous panel with unobserved common factors only}

We finally consider the following DGP, where only unobservable common factors are present:

$$
y_{i t}=\beta_{0 i}+\beta_{1 i} x_{1 i t}+\beta_{2 i} x_{2 i t}+\gamma_{i} f_{t}+\epsilon_{i t},
$$

and

$$
x_{i t}=a_{1 i}+a_{2 i} f_{t}+u_{i t},
$$


with $\beta_{0 i}=5+0.2 z_{i}$ and $z_{i}$ generated as i.i.d. $N(0,1)$; further, we also generate $a_{1 i}$ and $a_{2 i}$ as i.i.d. $N(0,1)$. We contrast the same estimators as in the previous subsection.

Bias and RMSEs are reported in Tables 7 and 8 respectively.

\section{[Insert Tables 7 and 8 somewhere here]}

Table 7 shows that all the estimators considered do not exhibit significant differences as far as the bias is concerned. Conversely, based on Table 8, the two-step procedure has a lower RMSE than the other procedures.

Finally, correlations between true and estimated factors, reported in Table 9 shows that in the case of Song's iterative procedure, the average correlation is always smaller than that obtained with the AMG procedure. The two average correlations converge only when $n$ becomes larger than 200. Finally, it should be noted how the Song's iterative procedure fail systematically to achieve convergence.

\section{[Insert Table 9 somewhere here]}

\subsection{Unknown number of Factors}

An important question is how robust are the various estimators to the knowledge of the true number of factors. We investigate the estimation of the number of factors problem via simulations. We extend the setup in Section 3.1 to the case of more factors, namely two factors. The factors $F_{t}=\left(F_{1 t}, F_{2 t}\right)$ are generated as i.i.d.N $(0,1)$. We estimate the number of factors by using the $I C_{p 2}$ criteria of Bai and $\mathrm{Ng}(2002) .{ }^{1}$ Table 10 reports the estimated number of factors averaged over 5, 000 replications. The first estimator, $\hat{r}_{A M G}$, is obtained from the residuals after the first stage CCEMG estimator. The estimator $\hat{r}_{\text {Song }}$ is obtained from the residuals of the Song's estimator. For the Song estimator we update the estimation of the factors at each iteration, until convergence of $\hat{\beta}$ according to the convergence criteria of Section 3.1. The estimate $\hat{r}_{A M G}$ stabilizes when $n, T>30$. On the contrary, the estimates of $r$ obtained with the Song's iterative procedure are more unstable and biased.

\footnotetext{
${ }^{1}$ We observe that changing the Bai and $\mathrm{Ng}(2002)$ criteria does not substantially alter the simulation results.
} 


\section{[Insert Table 10 somewhere here]}

The knowledge of the true number of factors does not play a very important role in improving the performance of the estimators, as it is evident from the bias and RMSE reported in Tables 11 and 12, respectively. Further, there is no clear indication that one of the estimators is more affected than others by the uncertainty in the number of the unknown factors.

\section{[Insert Table 11 and Table 12 somewhere here]}

\section{Conclusions}

This paper considers inference in a stationary panel model where slopes are allowed to be heterogeneous and common unknown factors are present. A two-stage estimator is proposed, based on the CCE estimator (Pesaran, 2006) in the first stage and on a similar approach to the Interactive Effect estimator (Bai, 2009) in the second stage. This affords the estimation of the common factors coefficients, and, it also yields an alternative estimator for the individual slopes and for the average slope. The properties of the slope estimators are analyzed. The finite sample properties are investigated by means of Monte Carlo simulations, under different data-generating processes. The results show that the two-step estimator proposed has remarkable properties when compared to the Bai (2009) iterative estimator and its extension to the case of heterogeneous panels provided by Song (2013). The IFE estimator is computationally more demanding and less accurate when we consider the fact that it fails to achieve convergence in a relevant number of cases. In conclusion, there is no clear advantage in finite sample in adopting the iterative procedure with respect to the much more reliable two-stage procedure proposed here. 


\section{Appendix A: Preliminary lemmas}

This section contains some preliminary Lemmas to prove Theorem 1. Lemmas 1-4 have been derived in Castagnetti, Rossi and Trapani (2012), and we refer to that paper for the proofs; Lemmas 5 and 6 are new, and the proofs are reported here. Their proofs are based on very similar arguments as in Castagnetti, Rossi, and Trapani (2012), and we only report the main passages when possible for the sake of a concise discussion.

Henceforth, we use the notation $\delta_{n T}=\min \{\sqrt{n}, \sqrt{T}\}$ and $\phi_{n T}=\min \{n, \sqrt{T}\}$. Note that, by equation (45) in Pesaran (2006, p.980)

$$
\begin{aligned}
\tilde{\beta}_{i}-\beta_{i} & =\left(X_{i}^{\prime} M_{F} X_{i}\right)^{-1}\left(X_{i}^{\prime} M_{F} \epsilon_{i}\right)+O_{p}\left(\frac{1}{n}\right)+O_{p}\left(\frac{1}{\sqrt{n T}}\right) \\
& =O_{p}\left(\frac{1}{\sqrt{T}}\right)+O_{p}\left(\frac{1}{n}\right)+O_{p}\left(\frac{1}{\sqrt{n T}}\right) .
\end{aligned}
$$

Both expressions (22) and (23) will be used frequently; similarly, we henceforth define $\Upsilon_{i}=$ $\left(X_{i}^{\prime} M_{F} X_{i}\right)^{-1}\left(X_{i}^{\prime} M_{F} \epsilon_{i}\right)$, so that we can write

$$
\tilde{\beta}_{i}-\beta_{i}=\Upsilon_{i}+\bar{\Upsilon}_{i}
$$

for every $i$; by construction, (23) yields $\bar{\Upsilon}_{i}=O_{p}\left(\frac{1}{n}\right)+O_{p}\left(\frac{1}{\sqrt{n T}}\right)$.

All proofs rely upon the decomposition - see Proposition A.1 in Bai (2009)

$$
\begin{aligned}
\hat{F}-F= & \frac{1}{n T} \sum_{j=1}^{n} X_{j}\left(\tilde{\beta}_{j}-\beta_{j}\right)\left(\tilde{\beta}_{j}-\beta_{j}\right)^{\prime} X_{j}^{\prime} \hat{F} \\
& -\frac{1}{n T} \sum_{j=1}^{n} X_{j}\left(\tilde{\beta}_{j}-\beta_{j}\right) \gamma_{j}^{\prime} F^{\prime} \hat{F}-\frac{1}{n T} \sum_{j=1}^{n} X_{j}\left(\tilde{\beta}_{j}-\beta_{j}\right) \epsilon_{j}^{\prime} \hat{F} \\
& -\frac{1}{n T} \sum_{j=1}^{n} F \gamma_{j}\left(\tilde{\beta}_{j}-\beta_{j}\right)^{\prime} X_{j}^{\prime} \hat{F}-\frac{1}{n T} \sum_{j=1}^{n} \epsilon_{j}\left(\tilde{\beta}_{j}-\beta_{j}\right)^{\prime} X_{j}^{\prime} \hat{F} \\
& +\frac{1}{n T} \sum_{j=1}^{n} F \gamma_{j} \epsilon_{j}^{\prime} \hat{F}+\frac{1}{n T} \sum_{j=1}^{n} \epsilon_{j} \gamma_{j}^{\prime} F^{\prime} \hat{F}+\frac{1}{n T} \sum_{j=1}^{n} \epsilon_{j} \epsilon_{j}^{\prime} \hat{F} \\
= & F 1+F 2+F 3+F 4+F 5+F 6+F 7+F 8 .
\end{aligned}
$$

For the sake of notational simplicity, we set rotation matrix $H$ in $\hat{F}-F H$ equal to the identity matrix. In view of (25), the only difference with Bai (2009) is the presence of the unit specific estimates, $\tilde{\beta}_{j}$. 
Lemma 1 Let Assumptions 1-4 hold. Then, as $(n, T) \rightarrow \infty$

$$
\begin{aligned}
X_{i}^{\prime}(\hat{F}-F) & =O_{p}\left(T \delta_{n T}^{-2}\right), \\
\hat{F}^{\prime}(\hat{F}-F) & =O_{p}\left(T \delta_{n T}^{-2}\right) .
\end{aligned}
$$

for all $i$.

Lemma 2 Let Assumptions $1-4$ hold. Then, as $(n, T) \rightarrow \infty$ it holds that, for every $i$

(i) $T^{-1} \epsilon_{i}^{\prime}(\hat{F}-F)=O_{p}\left(\delta_{n T}^{-2}\right)$;

(ii) $n^{-1 / 2} T^{-1} \sum_{i=1}^{n} \epsilon_{i}^{\prime}(\hat{F}-F)=O_{p}\left(n^{-1 / 2}\right)+O_{p}\left(T^{-1}\right)$.

Lemma 3 Let Assumptions 1-4 hold. Then it holds that, for every $i$

(i) $T^{-1} X_{i}^{\prime}(\hat{F}-F H)=O_{p}\left(\delta_{n T}^{-2}\right)$;

(ii) $T^{-1} F^{\prime}(\hat{F}-F H)=O_{p}\left(\delta_{n T}^{-2}\right)$;

(iii) $T^{-1}(\hat{F}-F H)^{\prime}(\hat{F}-F H)=O_{p}\left(\delta_{n T}^{-2}\right)$.

Lemma 4 Under Assumptions 1-4, it holds that, for every i, $E\left\|\tilde{\beta}_{i}-\beta_{i}\right\|^{r}=O\left(\phi_{n T}^{-r}\right)$, for any $r \leq 3$.

Lemma 5 Let Assumptions 1-4 hold. Then it holds that, for every $i$

(i) $T\left(X_{i}^{\prime} \hat{M}_{F} X_{i}\right)^{-1}=T\left(X_{i}^{\prime} M_{F} X_{i}\right)^{-1}+O_{p}\left(\delta_{n T}^{-2}\right)$;

(ii) $\frac{1}{n} \sum_{i=1}^{n}\left(X_{i}^{\prime} \hat{M}_{F} X_{i}\right)^{-1} X_{i}^{\prime} \hat{M}_{F} \epsilon_{i}=O_{p}\left(\delta_{n T}^{-2}\right)$.

Proof. Part (i) is similar to Lemma A.7 in Bai (2009). Consider $T^{-1} X_{i}^{\prime} \hat{M}_{F} X_{i}=T^{-1} X_{i}^{\prime} M_{F} X_{i}+$ $T^{-1} X_{i}^{\prime}\left(\hat{M}_{F}-M_{F}\right) X_{i}$. Using the definition of $\hat{M}_{F}$ and the identification restrictions $\hat{F}^{\prime} \hat{F}=T I_{k}$ and $F^{\prime} F=T I_{k}$, we have

$$
\begin{aligned}
& \frac{X_{i}^{\prime}\left(\hat{M}_{F}-M_{F}\right) X_{i}}{T}=\frac{X_{i}^{\prime} F}{T} \frac{(F-\hat{F})^{\prime} X_{i}}{T}+\frac{X_{i}^{\prime}(F-\hat{F})}{T} \frac{F^{\prime} X_{i}}{T} \\
& -\frac{X_{i}^{\prime}(\hat{F}-F)}{T} \frac{(\hat{F}-F)^{\prime} X_{i}}{T}=I+I^{\prime}-I I .
\end{aligned}
$$


Using Lemma 1(i), $I=O_{p}\left(\delta_{n T}^{-2}\right)$; similarly, $I I=O_{p}\left(\delta_{n T}^{-4}\right)$. Since $T^{-1} X_{i}^{\prime} M_{F} X_{i}$ is invertible by Assumption 4(i), part (i) follows.

We now turn to part (ii); using the short-hand notation $\Psi_{i}=T\left(X_{i}^{\prime} M_{F} X_{i}\right)^{-1}$ we have

$$
\begin{aligned}
& \frac{1}{n} \sum_{i=1}^{n}\left(X_{i}^{\prime} \hat{M}_{F} X_{i}\right)^{-1} X_{i}^{\prime} \hat{M}_{F} \epsilon_{i} \\
= & \frac{1}{n} \sum_{i=1}^{n} \Psi_{i} \frac{X_{i}^{\prime} \hat{M}_{F} \epsilon_{i}}{T}+\frac{1}{n} \sum_{i=1}^{n}\left[\left(\frac{X_{i}^{\prime} \hat{M}_{F} X_{i}}{T}\right)^{-1}-\left(\frac{X_{i}^{\prime} M_{F} X_{i}}{T}\right)^{-1}\right] \frac{X_{i}^{\prime} \hat{M}_{F} \epsilon_{i}}{T}+o_{p}(1) \\
= & \frac{1}{n} \sum_{i=1}^{n} \Psi_{i} \frac{X_{i}^{\prime} M_{F} \epsilon_{i}}{T}+\frac{1}{n} \sum_{i=1}^{n} \Psi_{i} \frac{X_{i}^{\prime}\left(\hat{M}_{F}-M_{F}\right) \epsilon_{i}}{T}+o_{p}(1)=I+I I+o_{p}(1),
\end{aligned}
$$

where the second equality follows from part (i) of the Lemma. Consider I; using Assumption 4

$$
E\left\|\frac{1}{n} \sum_{i=1}^{n} \Psi_{i} \frac{X_{i}^{\prime} M_{F} \epsilon_{i}}{T}\right\|^{2}=\frac{1}{n^{2}} \sum_{i=1}^{n} E\left\|\Psi_{i} \frac{X_{i}^{\prime} M_{F} \epsilon_{i}}{T}\right\|^{2} \leq \frac{1}{n^{2} T} \sum_{i=1}^{n} E\left\|\frac{X_{i}^{\prime} \epsilon_{i}}{\sqrt{T}}\right\|^{2}
$$

Note that

$E\left\|\frac{X_{j}^{\prime} \epsilon_{j}}{\sqrt{T}}\right\|^{2}=E\left\|\frac{1}{T} \sum_{t=1}^{T} \sum_{s=1}^{T} x_{j t} x_{j s}^{\prime} \epsilon_{j t} \epsilon_{j s}\right\| \leq \frac{1}{T} \sum_{t=1}^{T} \sum_{s=1}^{T} E\left\|x_{j t} x_{j s}^{\prime}\right\| E\left|\epsilon_{j t} \epsilon_{j s}\right| \leq M \frac{1}{T} \sum_{t=1}^{T} \sum_{s=1}^{T} E\left|\epsilon_{j t} \epsilon_{j s}\right| \leq M^{\prime}$

using, respectively, Assumptions 2(iii), 2(i) and 1(ii)(c). Thus, $I=O_{p}\left(n^{-1 / 2} T^{-1 / 2}\right)$. As far as $I I$ is concerned, note

$$
\begin{aligned}
-I I= & -\frac{1}{n} \sum_{i=1}^{n} \Psi_{i} \frac{X_{i}^{\prime}\left(\hat{M}_{F}-M_{F}\right) \epsilon_{i}}{T} \\
= & \frac{1}{n} \sum_{i=1}^{n} \Psi_{i}\left(\frac{X_{i}^{\prime} F}{T}\right) \frac{(\hat{F}-F)^{\prime} \epsilon_{i}}{T}+\frac{1}{n \sqrt{T}} \sum_{i=1}^{n} \Psi_{i} \frac{X_{i}^{\prime}(\hat{F}-F)}{T} \frac{F^{\prime} \epsilon_{i}}{\sqrt{T}} \\
& -\frac{1}{n} \sum_{i=1}^{n} \Psi_{i} \frac{X_{i}^{\prime}(\hat{F}-F)}{T} \frac{(\hat{F}-F)^{\prime} \epsilon_{i}}{T}=I I_{a}+I I_{b}-I I_{c} .
\end{aligned}
$$

Consider $I I_{a}$; this is bounded by $E\left[\left\|\frac{X_{i}^{\prime} F}{T}\right\|\left\|\frac{(\hat{F}-F)^{\prime} \epsilon_{i}}{T}\right\|\right]$. Using Lemma 2(i) and the same passages as above, $I I_{a}=O_{p}\left(\delta_{n T}^{-2}\right)$. Similarly, $\left\|I I_{b}\right\|$ is bounded by $T^{-1 / 2} E\left[\left\|\frac{X_{i}^{\prime}(\hat{F}-F)}{T}\right\|\left\|\frac{F^{\prime} \epsilon_{i}}{\sqrt{T}}\right\|\right]=$ $O_{p}\left(T^{-1 / 2} \delta_{n T}^{-2}\right)$. Similarly, $\left\|I I_{c}\right\|=O_{p}\left(\delta_{n T}^{-4}\right)$. Putting all together, $I I=O_{p}\left(\delta_{n T}^{-2}\right)$. Thus, $I+I I=$ $O_{p}\left(n^{-1 / 2} T^{-1 / 2}\right)+O_{p}\left(\delta_{n T}^{-2}\right)=O_{p}\left(\delta_{n T}^{-2}\right)$. QED

Lemma 6 Under Assumptions 1-4, it holds that $\frac{1}{n} \sum_{i=1}^{n}\left(X_{i}^{\prime} \hat{M}_{F} X_{i}\right)^{-1} X_{i}^{\prime} \hat{M}_{F} F \gamma_{i}=O_{p}\left(\delta_{n T}^{-2}\right)$. 
Proof. Using Lemma 5(i), $\frac{1}{n} \sum_{i=1}^{n}\left(X_{i}^{\prime} \hat{M}_{F} X_{i}\right)^{-1} X_{i}^{\prime} \hat{M}_{F} F \gamma_{i}=\frac{1}{n} \sum_{i=1}^{n}\left(X_{i}^{\prime} M_{F} X_{i}\right)^{-1} X_{i}^{\prime} \hat{M}_{F} F \gamma_{i}$ $+o_{p}(1)$. By the definition of $\hat{M}_{F}, \frac{1}{n} \sum_{i=1}^{n}\left(X_{i}^{\prime} \hat{M}_{F} X_{i}\right)^{-1} X_{i}^{\prime} \hat{M}_{F} F \gamma_{i}=\frac{1}{n} \sum_{i=1}^{n}\left(X_{i}^{\prime} \hat{M}_{F} X_{i}\right)^{-1} X_{i}^{\prime} \hat{M}_{F}(F-\hat{F}) \gamma_{i}$. Using (25), we obtain

$$
\begin{aligned}
& -\frac{1}{n} \sum_{i=1}^{n} \Psi_{i} \frac{X_{i}^{\prime} \hat{M}_{F}(F-\hat{F})}{T} \gamma_{i} \\
= & \frac{1}{n} \sum_{i=1}^{n} \Psi_{i} \frac{1}{n} \sum_{j=1}^{n} \frac{X_{i}^{\prime} \hat{M}_{F} X_{j}}{T}\left(\tilde{\beta}_{j}-\beta_{j}\right)\left(\tilde{\beta}_{j}-\beta_{j}\right)^{\prime} \frac{X_{j}^{\prime} \hat{F}}{T} \gamma_{i} \\
& -\frac{1}{n^{2} T} \sum_{i=1}^{n} \Psi_{i} X_{i}^{\prime} \hat{M}_{F} \sum_{j=1}^{n} X_{j}\left(\tilde{\beta}_{j}-\beta_{j}\right) \gamma_{j}^{\prime} \frac{F^{\prime} \hat{F}}{T} \gamma_{i} \\
& -\frac{1}{n^{2} T} \sum_{i=1}^{n} \Psi_{i} X_{i}^{\prime} \hat{M}_{F} \sum_{j=1}^{n} X_{j}\left(\tilde{\beta}_{j}-\beta_{j}\right) \frac{\epsilon_{j}^{\prime} \hat{F}}{T} \gamma_{i}-\frac{1}{n^{2} T} \sum_{i=1}^{n} \Psi_{i} X_{i}^{\prime} \hat{M}_{F} \sum_{j=1}^{n} F \gamma_{j}\left(\tilde{\beta}_{j}-\beta_{j}\right)^{\prime} \frac{X_{j}^{\prime} \hat{F}}{T} \gamma_{i} \\
& -\frac{1}{n^{2} T} \sum_{i=1}^{n} \Psi_{i} X_{i}^{\prime} \hat{M}_{F} \sum_{j=1}^{n} \epsilon_{j}\left(\tilde{\beta}_{j}-\beta_{j}\right)^{\prime} \frac{X_{j}^{\prime} \hat{F}}{T} \gamma_{i}+\frac{1}{n^{2} T} \sum_{i=1}^{n} \Psi_{i} X_{i}^{\prime} \hat{M}_{F} \sum_{j=1}^{n} F \gamma_{j} \frac{\epsilon_{j}^{\prime} \hat{F}}{T} \gamma_{i} \\
& +\frac{1}{n^{2} T} \sum_{i=1}^{n} \Psi_{i} X_{i}^{\prime} \hat{M}_{F} \sum_{j=1}^{n} \epsilon_{j} \gamma_{j}^{\prime} \frac{F^{\prime} \hat{F}}{T} \gamma_{i}+\frac{1}{n^{2} T} \sum_{i=1}^{n} \Psi_{i} X_{i}^{\prime} \hat{M}_{F} \sum_{j=1}^{n} \epsilon_{j} \frac{\epsilon_{j}^{\prime} \hat{F}}{T} \gamma_{i} \\
= & I-I I-I I I-I V-V+V I+V I I+V I I I .
\end{aligned}
$$

Consider $I$; we have

$I \leq E\left[\left\|\frac{X_{i}^{\prime} M_{F} X_{j}}{T}\right\|\left\|\frac{X_{j}^{\prime} \hat{F}}{T}\right\|\left\|\tilde{\beta}_{j}-\beta_{j}\right\|^{2}\right]+E\left[\left\|\frac{X_{i}^{\prime}\left(\hat{M}_{F}-M_{F}\right) X_{j}}{T}\right\|\left\|\frac{X_{j}^{\prime} \hat{F}}{T}\right\|\left\|\tilde{\beta}_{j}-\beta_{j}\right\|^{2}\right]=I_{a}+I_{b}$.

Hence, using a similar logic to the proofs in Castagnetti, Rossi, and Trapani (2012)

$$
\begin{aligned}
I_{a} & \leq\left[E\left(\left\|\tilde{\beta}_{j}-\beta_{j}\right\|^{2 p}\right)\right]^{1 / p}\left[E\left(\left\|\frac{X_{i}^{\prime} M_{F} X_{j}}{T}\right\|\left\|\frac{X_{j}^{\prime} \hat{F}}{T}\right\|\right)^{q}\right]^{1 / q} \\
& \leq\left[E\left\|\tilde{\beta}_{j}-\beta_{j}\right\|^{3}\right]^{2 / 3}\left[E\left\|\frac{X_{i}^{\prime} X_{j}}{T}\right\|^{6}\right]^{1 / 6}\left[E\left\|\frac{X_{j}^{\prime} \hat{F}}{T}\right\|^{6}\right]^{1 / 6},
\end{aligned}
$$

using Holder's inequality in the first line (with $p=\frac{3}{2}$ and $q=3$ ), and the Cauchy-Schwartz inequality in the second line. Lemma 4 yields that the first term is $O\left(\phi_{n T}^{-2}\right)$; Assumption 2 ensures that the second and the third term are both $O(1)$. As far as $I_{b}$ is concerned, by Lemma A.3(i)-(ii) in Bai (2009), $\left\|\hat{M}_{F}-M_{F}\right\|=o_{p}(1)$, and therefore $I_{b}$ is dominated. Thus, $I=O_{p}\left(\phi_{n T}^{-2}\right)$. Turning 
to $I I$, using (24)

$$
I I=\frac{1}{n^{2} T^{2}} \sum_{i=1}^{n} \Psi_{i} X_{i}^{\prime} M_{F}\left[\sum_{j=1}^{n} X_{j} \Upsilon_{j} \gamma_{j}^{\prime} F^{\prime} \hat{F} \gamma_{i}+\sum_{j=1}^{n} X_{j} \bar{\Upsilon}_{j} \gamma_{j}^{\prime} F^{\prime} \hat{F} \gamma_{i}\right]+o_{p}(1)=I I_{a}+I I_{b}+o_{p}(1)
$$

where the $o_{p}(1)$ term comes from $\left\|\hat{M}_{F}-M_{F}\right\|=o_{p}(1)$. Consider $I I_{a}$; this is bounded by

$$
\begin{aligned}
& \frac{1}{\sqrt{n T}}\left\|\frac{F^{\prime} \hat{F}}{T}\right\|\left[E\left\|\frac{X_{i}}{\sqrt{T}}\right\|^{2}\right]^{1 / 2}\left[E\left\|\frac{1}{\sqrt{n}} \sum_{j=1}^{n} X_{j}\left(\frac{X_{j}^{\prime} \bar{M}_{w} X_{j}}{T}\right)^{-1}\left(\frac{X_{j}^{\prime} \bar{M}_{w} \epsilon_{j}}{T}\right)\right\|^{2}\right]^{1 / 2} \\
= & O_{p}\left(\frac{1}{\sqrt{n T}}\right)
\end{aligned}
$$

based on the same passages as above. Turning to $I I_{b}$, this is bounded by $\left\|\frac{F^{\prime} \hat{F}}{T}\right\|\left[E\left\|\frac{X_{i}^{\prime} X_{j}}{T}\right\|^{2}\right]^{1 / 2}$ $\left[E\left\|\bar{\Upsilon}_{j}\right\|^{2}\right]^{1 / 2}$. Based on Lemma 3, Assumption 2, and (24), we have that this is $O_{p}\left(\frac{1}{n}\right)+$ $O_{p}\left(\frac{1}{\sqrt{n T}}\right)$. Hence, $I I=O_{p}\left(n^{-1 / 2} \delta_{n T}^{-1}\right)$. As far as $I I I$ is concerned, it is bounded by

$$
\begin{aligned}
& \frac{1}{n^{2} \sqrt{T}} \sum_{i=1}^{n} \sum_{j=1}^{n}\left\|\Psi_{i}\right\|\left\|\frac{X_{i}^{\prime} M_{F} X_{j}}{T}\right\|\left\|\tilde{\beta}_{j}-\beta_{j}\right\|\left\|\frac{\epsilon_{j}^{\prime} F}{\sqrt{T}}\right\| \\
& +\frac{1}{n^{2}} \sum_{i=1}^{n} \sum_{j=1}^{n}\left\|\Psi_{i}\right\|\left\|\frac{X_{i}^{\prime} M_{F} X_{j}}{T}\right\|\left\|\tilde{\beta}_{j}-\beta_{j}\right\|\left\|\frac{\epsilon_{j}^{\prime}(\hat{F}-F)}{T}\right\| \\
= & I I I_{a}+I I I_{b} .
\end{aligned}
$$

Term $I I I_{a}$ is bounded by $T^{-1 / 2} E\left[\left\|\frac{X_{i}^{\prime} X_{j}}{T}\right\|\left\|\tilde{\beta}_{j}-\beta_{j}\right\|\left\|\frac{\epsilon_{j}^{\prime} F}{\sqrt{T}}\right\|\right]$; we have

$$
I I I \leq\left[E\left\|\tilde{\beta}_{j}-\beta_{j}\right\|^{3}\right]^{2 / 3}\left[E\left\|\frac{X_{i}^{\prime} X_{j}}{T}\right\|^{6}\right]^{1 / 6}\left[E\left\|\frac{\epsilon_{j}^{\prime} F}{\sqrt{T}}\right\|^{6}\right]^{1 / 6}
$$

by a similar logic as above, $E\left\|\frac{\epsilon_{j}^{\prime} F}{\sqrt{T}}\right\|^{6}$ is bounded, and hence similar passages as above yield $I I I_{a}$ $=O_{p}\left(T^{-1 / 2} \phi_{n T}^{-1}\right)$. Similarly, by Lemma $2(i), I I I_{b}=O_{p}\left(\left\|\tilde{\beta}_{j}-\beta_{j}\right\|\right) O_{p}\left(\delta_{n T}^{-2}\right)$. This entails $I I I=$ $O_{p}\left(\phi_{n T}^{-1} \delta_{n T}^{-2}\right)$. As far as $I V$ and $V$ are concerned, they have the same order of magnitude as $I I$ and 
III respectively. Considering $V I$, it is bounded by

$$
\begin{aligned}
& \frac{1}{n \sqrt{n T}} \sum_{i=1}^{n}\left\|\Psi_{i}\right\|\left\|\frac{X_{i}^{\prime}\left(\hat{M}_{F}-M_{F}\right) F}{T}\right\|\left\|\frac{1}{\sqrt{n}} \sum_{j=1}^{n} \frac{\epsilon_{j}^{\prime} F}{\sqrt{T}}\right\| \\
& +\frac{1}{n \sqrt{n}} \sum_{i=1}^{n}\left\|\frac{X_{i}^{\prime}\left(\hat{M}_{F}-M_{F}\right) F}{T}\right\|\left\|\frac{1}{\sqrt{n}} \sum_{j=1}^{n} \frac{\epsilon_{j}^{\prime}(\hat{F}-F)}{T}\right\| \\
= & V I_{a}+V I_{b} .
\end{aligned}
$$

Note that $E\left\|\frac{X_{i}^{\prime} F}{T}\right\|$ can be shown to be bounded by using the Cauchy-Schwartz inequality and by using Assumption 2; further,

$$
\begin{aligned}
E\left\|\frac{1}{\sqrt{n}} \sum_{j=1}^{n} \frac{\epsilon_{j}^{\prime} F}{\sqrt{T}}\right\|^{2} & =E\left\|\frac{1}{n T} \sum_{j=1}^{n} \sum_{i=1}^{n} \sum_{t=1}^{T} \sum_{s=1}^{T} \epsilon_{i t} \epsilon_{j s} f_{t} f_{s}\right\| \\
& \leq M \frac{1}{n T} \sum_{j=1}^{n} \sum_{i=1}^{n} \sum_{t=1}^{T} \sum_{s=1}^{T} E\left\|\epsilon_{i t} \epsilon_{j s} f_{t} f_{s}\right\| \\
& \leq M^{\prime} \frac{1}{n T} \sum_{j=1}^{n} \sum_{i=1}^{n} \sum_{t=1}^{T} \sum_{s=1}^{T} E\left|\epsilon_{i t} \epsilon_{j s}\right| E\left\|f_{t} f_{s}\right\| \\
& \leq M^{\prime \prime} \frac{1}{n T} \sum_{j=1}^{n} \sum_{i=1}^{n} \sum_{t=1}^{T} \sum_{s=1}^{T} E\left|\epsilon_{i t} \epsilon_{j s}\right| \leq M^{\prime \prime \prime}
\end{aligned}
$$

using Assumption 2 and 1(ii)(d). Therefore, $V I_{a}$ is bounded by $\frac{1}{\sqrt{n T}}\left\|\hat{M}_{F}-M_{F}\right\| ;$ since $\left\|\hat{M}_{F}-M_{F}\right\|$ $=o_{p}(1), V I_{a}=o_{p}\left(n^{-1 / 2} T^{-1 / 2}\right)$. Turning to $V I_{b}$, note that $\left\|\frac{1}{\sqrt{n}} \sum_{j=1}^{n} \frac{\epsilon_{j}^{\prime}(\hat{F}-F)}{T}\right\|=O_{p}\left(\delta_{n T}^{-2}\right)$ by Lemma 2(ii); thus, $V I_{b}=o_{p}\left(n^{-1 / 2} \delta_{n T}^{-2}\right)$. Therefore, $V I=o_{p}\left(n^{-1 / 2} \delta_{n T}^{-1}\right)$. Turning to $V I I$, it is bounded by $n^{-1 / 2}\left\|\frac{F^{\prime} \hat{F}}{T}\right\| E\left\|n^{-1 / 2} \sum_{j=1}^{n} \Psi_{i} \frac{X_{i}^{\prime} \hat{M}_{F} \epsilon_{j}}{T}\right\|$; by Lemma 5 (ii), this entails that $V I I=$ $O_{p}\left(\delta_{n T}^{-2}\right)$. Finally, turning to VIII, this is bounded by

$$
\frac{1}{n^{2} \sqrt{T}} \sum_{i=1}^{n} \sum_{j=1}^{n}\left\|\Psi_{i} \frac{X_{i}^{\prime} \hat{M}_{F} \epsilon_{j}}{T}\right\|\left\|\frac{\epsilon_{j}^{\prime} F}{\sqrt{T}}\right\|+\frac{1}{n^{2}} \sum_{i=1}^{n} \sum_{j=1}^{n}\left\|\Psi_{i} \frac{X_{i}^{\prime} \hat{M}_{F} \epsilon_{j}}{T}\right\|\left\|\frac{\epsilon_{j}^{\prime}(\hat{F}-F)}{T}\right\|=V I I I_{a}+V I I I_{b} .
$$

As above, $V I I I_{a}$ is bounded by $T^{-1 / 2}\left[E\left\|\Psi_{i} \frac{X_{i}^{\prime} \hat{M}_{F} \epsilon_{j}}{T}\right\|^{2}\right]^{1 / 2}\left[E\left\|\frac{\epsilon_{j}^{\prime} F}{\sqrt{T}}\right\|^{2}\right]^{1 / 2}$. Given that $\Psi_{i} \frac{X_{i}^{\prime} M_{F} \epsilon_{j}}{T}=$ $O_{p}\left(T^{-1 / 2}\right)$, and that, using Lemma $3,\left\|\hat{M}_{F}-M_{F}\right\|=O_{p}\left(\delta_{n T}^{-2}\right)$, we have $\operatorname{VIII} I_{a}=O_{p}\left(T^{-1 / 2} \phi_{n T}^{-1}\right)$. Also, by Lemma $2(i), V I I I_{b}=O_{p}\left(\phi_{n T}^{-1}\right) O_{p}\left(\delta_{n T}^{-2}\right)$. Therefore, VIII $=O_{p}\left(\phi_{n T}^{-2}\right)$. Putting all together, the Lemma follows. QED 


\section{Appendix B}

Proof of Theorem 1. Consider (11) and (12). It holds that, by definition

$$
\begin{aligned}
\hat{\beta}^{A M G}-\beta & =\frac{1}{n} \sum_{i=1}^{n}\left(\beta_{i}-\beta\right)+\frac{1}{n} \sum_{i=1}^{n}\left(\hat{\beta}_{i}-\beta_{i}\right) \\
& =\frac{1}{n} \sum_{i=1}^{n}\left(\beta_{i}-\beta\right)+\frac{1}{n} \sum_{i=1}^{n}\left(X_{i}^{\prime} \hat{M}_{F} X_{i}\right)^{-1}\left(X_{i}^{\prime} \hat{M}_{F} \epsilon_{i}+X_{i}^{\prime} \hat{M}_{F} F \gamma_{i}\right)
\end{aligned}
$$

and

$$
\hat{\beta}^{A P}-\beta=\left[\frac{1}{n} \sum_{i=1}^{n} X_{i}^{\prime} \hat{M}_{F} X_{i}\right]^{-1} \times\left[\frac{1}{n} \sum_{i=1}^{n} X_{i}^{\prime} \hat{M}_{F} X_{i}\left(\beta_{i}-\beta\right)+\frac{1}{n} \sum_{i=1}^{n} X_{i}^{\prime} \hat{M}_{F} \epsilon_{i}+\frac{1}{n} \sum_{i=1}^{n} X_{i}^{\prime} \hat{M}_{F} F \gamma_{i}\right] .
$$

Equation (11) follows immediately from Lemmas 3 and 5, and by noting that, using Assumption 3,

$$
E\left\|\frac{1}{n} \sum_{i=1}^{n}\left(\beta_{i}-\beta\right)\right\|^{2}=\frac{1}{n^{2}} \sum_{i=1}^{n} E\left\|\left(\beta_{i}-\beta\right)\right\|^{2}=O\left(\frac{1}{n}\right)
$$

and

$$
E\left\|\frac{1}{n} \sum_{i=1}^{n} X_{i}^{\prime} \hat{M}_{F} X_{i}\left(\beta_{i}-\beta\right)\right\|^{2}=\frac{1}{n^{2}} \sum_{i=1}^{n} E\left\|X_{i}^{\prime} \hat{M}_{F} X_{i}\right\|^{2} E\left\|\left(\beta_{i}-\beta\right)\right\|^{2}=O\left(\frac{1}{n}\right) .
$$

Consider now (12). The term that dominates in (28) is $\frac{1}{n} \sum_{i=1}^{n}\left(\beta_{i}-\beta\right)$; under Assumption 3, a Central Limit Theorem (CLT) holds, so that (12) follows immediately. As far as (13) is concerned, in (29) the term that dominates is $\left[\frac{1}{n} \sum_{i=1}^{n} X_{i}^{\prime} \hat{M}_{F} X_{i}\right]^{-1}\left[\frac{1}{n} \sum_{i=1}^{n} X_{i}^{\prime} \hat{M}_{F} X_{i}\left(\beta_{i}-\beta\right)\right]$. Consider the denominator; from the above we have

$$
\frac{1}{n} \sum_{i=1}^{n} X_{i}^{\prime} \hat{M}_{F} X_{i}=\frac{1}{n} \sum_{i=1}^{n} X_{i}^{\prime} M_{F} X_{i}+o_{p}(1)
$$

note that the $X_{i}^{\prime} M_{F} X_{i}$ s are a conditionally independent sequence with finite second moment indeed, by Assumption 2, the sequence has moments up to the 6th. Hence a conditional Law of Large Numbers can be applied (see e.g. Rao (2009)), and $\frac{1}{n} \sum_{i=1}^{n} X_{i}^{\prime} M_{F} X_{i} \stackrel{d}{\rightarrow} \frac{1}{n} \sum_{i=1}^{n} E\left(X_{i}^{\prime} M_{F} X_{i}\right)$, where $E$ represents the expected value operator conditional upon the $\sigma$-field $\left\{f_{t}\right\}_{t=1}^{T}$. As far as the numerator is concerned, note that $X_{i}^{\prime} \hat{M}_{F} X_{i}\left(\beta_{i}-\beta\right)=X_{i}^{\prime} M_{F} X_{i}\left(\beta_{i}-\beta\right)+o_{p}(1)$; further, the sequence $X_{i}^{\prime} M_{F} X_{i}\left(\beta_{i}-\beta\right)$ is, conditional upon $\left\{f_{t}\right\}_{t=1}^{T}$, independent across $i$. A Lyapunov condition can be shown to hold, with $E\left\|X_{i}^{\prime} M_{F} X_{i}\left(\beta_{i}-\beta\right)\right\|^{2+\delta} \leq E\left\|X_{i}^{\prime} X_{i}\right\|^{2+\delta} E\left\|\beta_{i}-\beta\right\|^{2+\delta}$, on 
account of Assumptions 2 and 3. Thus, the conditional CLT (Rao, 2009) yields

$$
\frac{1}{\sqrt{n}} \sum_{i=1}^{n} X_{i}^{\prime} M_{F} X_{i}\left(\beta_{i}-\beta\right) \stackrel{d}{\rightarrow} V^{1 / 2} \times Z,
$$

with $Z \sim N\left(0, I_{k}\right)$ independent of $\left\{f_{t}\right\}_{t=1}^{T}$ and

$$
\begin{aligned}
V & =\lim _{n, T \rightarrow \infty} E\left[\frac{1}{n} \sum_{i=1}^{n} \sum_{j=1}^{n} \frac{X_{i}^{\prime} M_{F} X_{i}}{T}\left(\beta_{i}-\beta\right)\left(\beta_{j}-\beta\right)^{\prime} \frac{X_{j}^{\prime} M_{F} X_{j}}{T}\right] \\
& =\lim _{n, T \rightarrow \infty} E\left[\frac{1}{n} \sum_{i=1}^{n} \frac{X_{i}^{\prime} M_{F} X_{i}}{T}\left(\beta_{i}-\beta\right)\left(\beta_{i}-\beta\right)^{\prime} \frac{X_{i}^{\prime} M_{F} X_{i}}{T}\right] \\
& =\lim _{n, T \rightarrow \infty} E\left[\frac{1}{n} \sum_{i=1}^{n} \frac{X_{i}^{\prime} M_{F} X_{i}}{T} \Omega_{v} \frac{X_{i}^{\prime} M_{F} X_{i}}{T}\right] .
\end{aligned}
$$

Putting all together, (13) follows.

Finally, note that, when $\beta_{i}=\beta$ (and, therefore, $\Omega_{v}=0$ ) for all $i, \hat{\beta}^{A M G}-\beta$ reduces to $n^{-1} \sum_{i=1}^{n}\left(X_{i}^{\prime} \hat{M}_{F} X_{i}\right)^{-1} X_{i}^{\prime} \hat{M}_{F} \epsilon_{i}+n^{-1} \sum_{i=1}^{n}\left(X_{i}^{\prime} \hat{M}_{F} X_{i}\right)^{-1} X_{i}^{\prime} \hat{M}_{F} F \gamma_{i}$, which as mentioned above is $O_{p}\left(\delta_{n T}^{-2}\right)$. The same passages yield the same result for $\hat{\beta}^{A P}$. 


\begin{tabular}{rr|cccc}
\hline$n$ & $T$ & $\operatorname{Corr}\left(F_{t}, \hat{F}_{I F E, t}\right)$ & $\operatorname{Corr}\left(F_{t}, \hat{F}_{A P, t}\right)$ & iter & fail \\
\hline 10 & 10 & 0.5939 & 0.6463 & 43.102 & 58 \\
10 & 30 & 0.6878 & 0.7194 & 41.775 & 32 \\
10 & 50 & 0.7354 & 0.7564 & 40.756 & 12 \\
10 & 100 & 0.7744 & 0.7820 & 44.141 & 4 \\
10 & 200 & 0.7974 & 0.7922 & 45.345 & 3 \\
30 & 10 & 0.7900 & 0.7815 & 41.345 & 29 \\
30 & 30 & 0.9017 & 0.8970 & 42.350 & 0 \\
30 & 50 & 0.9196 & 0.9131 & 43.200 & 0 \\
30 & 100 & 0.9287 & 0.9231 & 43.013 & 0 \\
30 & 200 & 0.9323 & 0.9275 & 43.452 & 0 \\
50 & 10 & 0.8679 & 0.8421 & 42.504 & 24 \\
50 & 30 & 0.9458 & 0.9321 & 44.192 & 0 \\
50 & 50 & 0.9536 & 0.9443 & 43.239 & 0 \\
50 & 100 & 0.9574 & 0.9521 & 43.231 & 0 \\
50 & 200 & 0.9596 & 0.9561 & 42.895 & 0 \\
100 & 10 & 0.9419 & 0.8957 & 43.584 & 2 \\
100 & 30 & 0.9745 & 0.9576 & 43.817 & 0 \\
100 & 50 & 0.9773 & 0.9671 & 42.990 & 0 \\
100 & 100 & 0.9788 & 0.9737 & 42.678 & 0 \\
100 & 200 & 0.9796 & 0.9768 & 42.192 & 0 \\
200 & 10 & 0.9732 & 0.9183 & 45.069 & 1 \\
200 & 30 & 0.9876 & 0.9702 & 43.386 & 0 \\
200 & 50 & 0.9888 & 0.9784 & 43.043 & 0 \\
200 & 100 & 0.9894 & 0.9843 & 42.290 & 0 \\
200 & 200 & 0.9898 & 0.9872 & 42.080 & 0 \\
\hline & & & & & \\
& & & & &
\end{tabular}

Table 1: Average correlation coefficients between $\left\{F_{t}\right\}_{t=1}^{T}$ and $\left\{\hat{F}_{I F E, t}\right\}_{t=1}^{T}$ and $\left\{\hat{F}_{A P, t}\right\}_{t=1}^{T}$ in the case of homogeneous slope. Iter and fail indicate the average number of iterations and the number of failures (lack of convergence) of the iterative process for the estimation method of Bai (2009), respectively. The DGP is in (15)-(17). 


\begin{tabular}{rr|rrrrrrrr}
\hline & & \multicolumn{2}{|c}{ IFE } & \multicolumn{2}{c}{ CCEP } & \multicolumn{2}{c}{ Inf Pooled } & \multicolumn{2}{c}{ AP } \\
\hline$n$ & $T$ & $\beta_{1}$ & $\beta_{2}$ & $\beta_{1}$ & $\beta_{2}$ & \multicolumn{1}{c}{$\beta_{1}$} & \multicolumn{1}{c}{$\beta_{2}$} & $\beta_{1}$ & $\beta_{2}$ \\
\hline 10 & 10 & 0.0940 & 0.0917 & 0.0548 & 0.0473 & -0.0003 & -0.0010 & 0.0665 & 0.0658 \\
10 & 30 & 0.0606 & 0.0585 & 0.0515 & 0.0481 & 0.0010 & -0.0011 & 0.0409 & 0.0388 \\
10 & 50 & 0.0440 & 0.0461 & 0.0500 & 0.0509 & -0.0011 & -0.0003 & 0.0296 & 0.0310 \\
10 & 100 & 0.0317 & 0.0302 & 0.0526 & 0.0504 & 0.0007 & -0.0009 & 0.0242 & 0.0229 \\
10 & 200 & 0.0166 & 0.0173 & 0.0522 & 0.0526 & 0.0001 & 0.0003 & 0.0189 & 0.0196 \\
30 & 10 & 0.0655 & 0.0606 & 0.0201 & 0.0142 & 0.0009 & -0.0021 & 0.0517 & 0.0470 \\
30 & 30 & 0.0268 & 0.0258 & 0.0175 & 0.0163 & 0.0003 & -0.0011 & 0.0175 & 0.0163 \\
30 & 50 & 0.0155 & 0.0141 & 0.0183 & 0.0167 & 0.0010 & -0.0003 & 0.0118 & 0.0103 \\
30 & 100 & 0.0083 & 0.0072 & 0.0179 & 0.0167 & 0.0005 & -0.0004 & 0.0082 & 0.0071 \\
30 & 200 & 0.0037 & 0.0040 & 0.0173 & 0.0175 & 0.0001 & 0.0004 & 0.0047 & 0.0050 \\
50 & 10 & 0.0512 & 0.0536 & 0.0082 & 0.0150 & -0.0004 & 0.0026 & 0.0412 & 0.0458 \\
50 & 30 & 0.0150 & 0.0152 & 0.0097 & 0.0103 & -0.0004 & -0.0002 & 0.0130 & 0.0133 \\
50 & 50 & 0.0083 & 0.0079 & 0.0103 & 0.0094 & 0.0002 & -0.0003 & 0.0081 & 0.0078 \\
50 & 100 & 0.0036 & 0.0041 & 0.0098 & 0.0103 & -0.0003 & 0.0002 & 0.0045 & 0.0050 \\
50 & 200 & 0.0021 & 0.0025 & 0.0103 & 0.0107 & -0.0001 & 0.0004 & 0.0027 & 0.0031 \\
100 & 10 & 0.0287 & 0.0299 & 0.0055 & 0.0042 & -0.0007 & 0.0006 & 0.0331 & 0.0348 \\
100 & 30 & 0.0066 & 0.0078 & 0.0040 & 0.0054 & -0.0005 & 0.0008 & 0.0094 & 0.0106 \\
100 & 50 & 0.0039 & 0.0036 & 0.0051 & 0.0045 & 0.0000 & -0.0002 & 0.0067 & 0.0064 \\
100 & 100 & 0.0023 & 0.0022 & 0.0053 & 0.0052 & -0.0002 & -0.0002 & 0.0035 & 0.0034 \\
100 & 200 & 0.0012 & 0.0011 & 0.0051 & 0.0050 & 0.0000 & -0.0001 & 0.0017 & 0.0017 \\
200 & 10 & 0.0160 & 0.0163 & 0.0019 & 0.0023 & -0.0002 & 0.0002 & 0.0298 & 0.0303 \\
200 & 30 & 0.0040 & 0.0035 & 0.0027 & 0.0023 & 0.0001 & -0.0003 & 0.0090 & 0.0087 \\
200 & 50 & 0.0018 & 0.0025 & 0.0023 & 0.0029 & -0.0002 & 0.0005 & 0.0054 & 0.0062 \\
200 & 100 & 0.0010 & 0.0013 & 0.0024 & 0.0028 & 0.0000 & 0.0003 & 0.0020 & 0.0024 \\
200 & 200 & 0.0007 & 0.0007 & 0.0026 & 0.0026 & 0.0001 & 0.0001 & 0.0012 & 0.0012 \\
\hline
\end{tabular}

Table 2: Bias of $\beta$ estimators in the case of homogeneous slope. The DGP is in (15)-(15). IFE is the Interactive Fixed Effects estimator by Bai (2009), CCEP is the Pooled CCE estimator by Pesaran (2006), Inf Pooled is the infeasible pooled estimator obtained with pooling and unobserved common factors. $A P$ is the Augmented Pooled estimator. 


\begin{tabular}{|c|c|c|c|c|c|c|c|c|c|}
\hline \multirow[b]{2}{*}{$n$} & \multirow[b]{2}{*}{$T$} & \multicolumn{2}{|c|}{ IFE } & \multicolumn{2}{|c|}{ CCEP } & \multicolumn{2}{|c|}{ Inf Pooled } & \multicolumn{2}{|c|}{ AP } \\
\hline & & $\beta_{1}$ & $\beta_{2}$ & $\beta_{1}$ & $\beta_{2}$ & $\beta_{1}$ & $\beta_{2}$ & $\beta_{1}$ & $\beta_{2}$ \\
\hline 10 & 10 & 0.2563 & 0.2469 & 0.3054 & 0.2911 & 0.1767 & 0.1751 & 0.2182 & 0.2090 \\
\hline 10 & 30 & 0.1594 & 0.1594 & 0.1499 & 0.1467 & 0.0968 & 0.1022 & 0.1225 & 0.1253 \\
\hline 10 & 50 & 0.1245 & 0.1281 & 0.1123 & 0.1139 & 0.0754 & 0.0723 & 0.0919 & 0.0916 \\
\hline 10 & 100 & 0.0940 & 0.0965 & 0.0945 & 0.0939 & 0.0508 & 0.0537 & 0.0650 & 0.0668 \\
\hline 10 & 200 & 0.0668 & 0.0669 & 0.0766 & 0.0785 & 0.0366 & 0.0378 & 0.0464 & 0.0485 \\
\hline 30 & 10 & 0.1584 & 0.1558 & 0.1691 & 0.1675 & 0.1005 & 0.0981 & 0.1378 & 0.1391 \\
\hline 30 & 30 & 0.0894 & 0.0877 & 0.0752 & 0.0767 & 0.0550 & 0.0570 & 0.0762 & 0.0787 \\
\hline 30 & 50 & 0.0657 & 0.0637 & 0.0581 & 0.0592 & 0.0434 & 0.0433 & 0.0601 & 0.0610 \\
\hline 30 & 100 & 0.0446 & 0.0419 & 0.0436 & 0.0429 & 0.0311 & 0.0306 & 0.0402 & 0.0424 \\
\hline 30 & 200 & 0.0282 & 0.0288 & 0.0332 & 0.0332 & 0.0217 & 0.0214 & 0.0302 & 0.0307 \\
\hline 50 & 10 & 0.1260 & 0.1310 & 0.1303 & 0.1277 & 0.0736 & 0.0749 & 0.1136 & 0.1194 \\
\hline 50 & 30 & 0.0625 & 0.0651 & 0.0546 & 0.0582 & 0.0403 & 0.0430 & 0.0661 & 0.0700 \\
\hline 50 & 50 & 0.0477 & 0.0478 & 0.0438 & 0.0444 & 0.0323 & 0.0348 & 0.0524 & 0.0530 \\
\hline 50 & 100 & 0.0313 & 0.0314 & 0.0316 & 0.0308 & 0.0234 & 0.0229 & 0.0383 & 0.0376 \\
\hline 50 & 200 & 0.0216 & 0.0218 & 0.0230 & 0.0234 & 0.0166 & 0.0167 & 0.0270 & 0.0271 \\
\hline 100 & 10 & 0.0963 & 0.0943 & 0.0941 & 0.0898 & 0.0561 & 0.0533 & 0.1044 & 0.1067 \\
\hline 100 & 30 & 0.0436 & 0.0438 & 0.0407 & 0.0402 & 0.0318 & 0.0313 & 0.0644 & 0.0628 \\
\hline 100 & 50 & 0.0300 & 0.0310 & 0.0297 & 0.0305 & 0.0232 & 0.0228 & 0.0489 & 0.0501 \\
\hline 100 & 100 & 0.0214 & 0.0214 & 0.0211 & 0.0210 & 0.0167 & 0.0171 & 0.0351 & 0.0357 \\
\hline 100 & 200 & 0.0147 & 0.0144 & 0.0150 & 0.0145 & 0.0119 & 0.0111 & 0.0252 & 0.0250 \\
\hline 200 & 10 & 0.0668 & 0.0688 & 0.0606 & 0.0610 & 0.0375 & 0.0378 & 0.0990 & 0.0984 \\
\hline 200 & 30 & 0.0289 & 0.0291 & 0.0285 & 0.0289 & 0.0218 & 0.0218 & 0.0609 & 0.0613 \\
\hline 200 & 50 & 0.0214 & 0.0209 & 0.0210 & 0.0208 & 0.0164 & 0.0162 & 0.0484 & 0.0490 \\
\hline 200 & 100 & 0.0147 & 0.0150 & 0.0145 & 0.0153 & 0.0116 & 0.0117 & 0.0343 & 0.0345 \\
\hline 200 & 200 & 0.0102 & 0.0103 & 0.0105 & 0.0104 & 0.0082 & 0.0083 & 0.0242 & 0.0249 \\
\hline
\end{tabular}

Table 3: RMSE of $\beta$ estimators in the case of homogeneous slope. The DGP is in (15)-(15). IFE is the Interactive Fixed Effects estimator by Bai (2009), CCEP is the Pooled CCE estimator by Pesaran (2006), Inf Pooled is the infeasible pooled estimator obtained with pooling and unobserved common factors. AP is the Augmented Pooled estimator. 


\begin{tabular}{rrrrrrrrrr}
\hline & \multicolumn{1}{c}{ Inf } & MG & \multicolumn{2}{c}{ CCEMG } & \multicolumn{1}{c}{ AMG } & \multicolumn{2}{c}{ Song } \\
\hline$n$ & $T$ & \multicolumn{1}{c}{$\beta_{1}$} & \multicolumn{1}{c}{$\beta_{2}$} & \multicolumn{1}{c}{$\beta_{1}$} & \multicolumn{1}{c}{$\beta_{2}$} & \multicolumn{1}{c}{$\beta_{1}$} & \multicolumn{1}{c}{$\beta_{1}$} & $\beta_{2}$ \\
\hline 10 & 10 & 0.0327 & 0.1679 & 0.0763 & 0.2155 & 0.0778 & 0.2120 & 0.1374 & 0.2583 \\
10 & 30 & -0.1060 & 0.0210 & -0.0626 & 0.0648 & -0.0740 & 0.0516 & -0.0282 & 0.0979 \\
10 & 50 & -0.0183 & -0.0250 & 0.0275 & 0.0197 & 0.0092 & 0.0004 & 0.0513 & 0.0430 \\
10 & 100 & 0.1007 & -0.0208 & 0.1446 & 0.0235 & 0.1226 & 0.0016 & 0.1551 & 0.0349 \\
10 & 200 & 0.0543 & -0.0062 & 0.0983 & 0.0377 & 0.0752 & 0.0143 & 0.0944 & 0.0336 \\
30 & 10 & -0.0141 & 0.0104 & 0.0016 & 0.0221 & 0.0122 & 0.0373 & 0.0863 & 0.1109 \\
30 & 30 & -0.0202 & -0.0463 & -0.0032 & -0.0305 & -0.0087 & -0.0344 & 0.0389 & 0.0118 \\
30 & 50 & -0.0362 & 0.0194 & -0.0198 & 0.0357 & -0.0268 & 0.0287 & 0.0078 & 0.0632 \\
30 & 100 & 0.0127 & 0.0171 & 0.0290 & 0.0333 & 0.0190 & 0.0234 & 0.0388 & 0.0433 \\
30 & 200 & 0.0033 & 0.0013 & 0.0196 & 0.0177 & 0.0087 & 0.0067 & 0.0173 & 0.0152 \\
50 & 10 & -0.0079 & 0.0231 & 0.0048 & 0.0317 & 0.0138 & 0.0437 & 0.0870 & 0.1137 \\
50 & 30 & 0.0218 & -0.0217 & 0.0316 & -0.0119 & 0.0296 & -0.0139 & 0.0681 & 0.0248 \\
50 & 50 & 0.0071 & -0.0281 & 0.0170 & -0.0181 & 0.0124 & -0.0228 & 0.0377 & 0.0023 \\
50 & 100 & 0.0129 & 0.0231 & 0.0227 & 0.0328 & 0.0165 & 0.0268 & 0.0291 & 0.0394 \\
50 & 200 & 0.0384 & 0.0223 & 0.0483 & 0.0322 & 0.0412 & 0.0252 & 0.0463 & 0.0304 \\
100 & 10 & -0.0009 & -0.0320 & 0.0047 & -0.0251 & 0.0141 & -0.0174 & 0.0829 & 0.0523 \\
100 & 30 & -0.0436 & 0.0376 & -0.0385 & 0.0426 & -0.0395 & 0.0417 & -0.0142 & 0.0670 \\
100 & 50 & -0.0261 & 0.0216 & -0.0213 & 0.0264 & -0.0232 & 0.0244 & -0.0088 & 0.0389 \\
100 & 100 & -0.0077 & -0.0145 & -0.0027 & -0.0096 & -0.0059 & -0.0127 & 0.0006 & -0.0062 \\
100 & 200 & 0.0175 & -0.0098 & 0.0225 & -0.0048 & 0.0187 & -0.0085 & 0.0215 & -0.0058 \\
200 & 10 & 0.0173 & -0.0028 & 0.0195 & 0.0019 & 0.0249 & 0.0056 & 0.0883 & 0.0685 \\
200 & 30 & -0.0223 & -0.0030 & -0.0195 & -0.0006 & -0.0197 & -0.0006 & -0.0057 & 0.0136 \\
200 & 50 & -0.0027 & 0.0016 & -0.0001 & 0.0041 & -0.0013 & 0.0030 & 0.0062 & 0.0104 \\
200 & 100 & 0.0076 & 0.0222 & 0.0101 & 0.0247 & 0.0084 & 0.0230 & 0.0117 & 0.0264 \\
200 & 200 & -0.0011 & 0.0051 & 0.0013 & 0.0076 & -0.0006 & 0.0057 & 0.0008 & 0.0071 \\
\hline & & & & & & & & & \\
\hline
\end{tabular}

Table 4: Bias of $\beta_{i}$ estimators in the case of heterogeneous slope, see (18) and (19). Inf $M G$ is the mean group estimators obtained with unobserved common factors. CCEMG is the mean group CCE estimator by Pesaran (2006). $A M G$ is the mean group estimator in (8). Song is the Interactive Fixed Effects estimator by Bai (2009) extended to the heterogenous case by Song (2013). 


\begin{tabular}{rrcccccccc}
\hline \multicolumn{1}{c}{ Inf MG } & \multicolumn{2}{c}{ CCEMG } & \multicolumn{2}{c}{ AMG } & \multicolumn{2}{c}{ Song } \\
\hline$n$ & $T$ & $\beta_{1}$ & $\beta_{2}$ & $\beta_{1}$ & $\beta_{2}$ & $\beta_{1}$ & $\beta_{2}$ & $\beta_{1}$ & $\beta_{2}$ \\
\hline 10 & 10 & 0.3190 & 0.3203 & 0.4517 & 0.4626 & 0.3462 & 0.3453 & 0.4861 & 0.4880 \\
10 & 30 & 0.1283 & 0.1253 & 0.1463 & 0.1473 & 0.1439 & 0.1450 & 0.1908 & 0.1853 \\
10 & 50 & 0.0943 & 0.0962 & 0.1131 & 0.1158 & 0.1087 & 0.1111 & 0.1471 & 0.1505 \\
10 & 100 & 0.0649 & 0.0658 & 0.0859 & 0.0857 & 0.0769 & 0.0767 & 0.1129 & 0.1127 \\
10 & 200 & 0.0450 & 0.0453 & 0.0707 & 0.0704 & 0.0565 & 0.0562 & 0.0863 & 0.0849 \\
30 & 10 & 0.1846 & 0.1840 & 0.2583 & 0.2573 & 0.1923 & 0.1952 & 0.2363 & 0.2384 \\
30 & 30 & 0.0751 & 0.0754 & 0.0812 & 0.0802 & 0.0808 & 0.0799 & 0.1057 & 0.1052 \\
30 & 50 & 0.0556 & 0.0556 & 0.0602 & 0.0608 & 0.0600 & 0.0600 & 0.0800 & 0.0808 \\
30 & 100 & 0.0376 & 0.0374 & 0.0426 & 0.0423 & 0.0405 & 0.0402 & 0.0538 & 0.0542 \\
30 & 200 & 0.0264 & 0.0264 & 0.0326 & 0.0318 & 0.0290 & 0.0288 & 0.0357 & 0.0358 \\
50 & 10 & 0.1401 & 0.1438 & 0.1992 & 0.1990 & 0.1476 & 0.1494 & 0.1857 & 0.1881 \\
50 & 30 & 0.0573 & 0.0565 & 0.0608 & 0.0603 & 0.0601 & 0.0590 & 0.0805 & 0.0798 \\
50 & 50 & 0.0426 & 0.0427 & 0.0451 & 0.0447 & 0.0446 & 0.0448 & 0.0582 & 0.0580 \\
50 & 100 & 0.0291 & 0.0293 & 0.0314 & 0.0317 & 0.0304 & 0.0308 & 0.0377 & 0.0375 \\
50 & 200 & 0.0199 & 0.0206 & 0.0229 & 0.0233 & 0.0212 & 0.0218 & 0.0238 & 0.0242 \\
100 & 10 & 0.1019 & 0.1003 & 0.1453 & 0.1402 & 0.1067 & 0.1059 & 0.1355 & 0.1369 \\
100 & 30 & 0.0411 & 0.0405 & 0.0434 & 0.0427 & 0.0426 & 0.0419 & 0.0549 & 0.0543 \\
100 & 50 & 0.0305 & 0.0304 & 0.0317 & 0.0316 & 0.0316 & 0.0314 & 0.0379 & 0.0379 \\
100 & 100 & 0.0206 & 0.0206 & 0.0215 & 0.0215 & 0.0211 & 0.0213 & 0.0236 & 0.0237 \\
100 & 200 & 0.0144 & 0.0144 & 0.0154 & 0.0154 & 0.0148 & 0.0149 & 0.0155 & 0.0156 \\
200 & 10 & 0.0721 & 0.0708 & 0.0992 & 0.1005 & 0.0742 & 0.0731 & 0.1043 & 0.1037 \\
200 & 30 & 0.0289 & 0.0292 & 0.0307 & 0.0304 & 0.0296 & 0.0296 & 0.0357 & 0.0353 \\
200 & 50 & 0.0211 & 0.0210 & 0.0218 & 0.0217 & 0.0215 & 0.0213 & 0.0239 & 0.0238 \\
200 & 100 & 0.0145 & 0.0148 & 0.0149 & 0.0151 & 0.0148 & 0.0150 & 0.0156 & 0.0158 \\
200 & 200 & 0.0102 & 0.0101 & 0.0106 & 0.0105 & 0.0104 & 0.0102 & 0.0106 & 0.0105 \\
\hline & & & & & & & & & \\
\hline
\end{tabular}

Table 5: RMSE of $\beta_{i}$ estimators in the case of heterogeneous slope, see (18) and (19). Inf $M G$ is the mean group estimators obtained with unobserved common factors. CCEMG is the mean group CCE estimator by Pesaran (2006). $A M G$ is the mean group estimator in (8). Song is the Interactive Fixed Effects estimator by Bai (2009) extended to the heterogenous case by Song (2013). 


\begin{tabular}{rr|cccc}
\hline$n$ & $T$ & $\operatorname{Corr}\left(F_{t}, \hat{F}_{\text {Song, } t}\right)$ & $\operatorname{Corr}\left(F_{t}, \hat{F}_{A M G, t}\right)$ & iter & fail \\
\hline 10 & 10 & 0.490 & 0.687 & 145.659 & 2300 \\
10 & 30 & 0.592 & 0.752 & 92.385 & 884 \\
10 & 50 & 0.646 & 0.781 & 91.724 & 860 \\
10 & 100 & 0.699 & 0.803 & 95.889 & 876 \\
10 & 200 & 0.739 & 0.811 & 104.351 & 1028 \\
30 & 10 & 0.562 & 0.816 & 155.686 & 2487 \\
30 & 30 & 0.791 & 0.908 & 126.797 & 1438 \\
30 & 50 & 0.852 & 0.921 & 133.215 & 1463 \\
30 & 100 & 0.893 & 0.931 & 142.441 & 1590 \\
30 & 200 & 0.915 & 0.935 & 147.912 & 1506 \\
50 & 10 & 0.601 & 0.856 & 168.865 & 3020 \\
50 & 30 & 0.867 & 0.939 & 152.717 & 2157 \\
50 & 50 & 0.911 & 0.949 & 159.180 & 2173 \\
50 & 100 & 0.938 & 0.957 & 163.199 & 1987 \\
50 & 200 & 0.950 & 0.960 & 161.316 & 1655 \\
100 & 10 & 0.679 & 0.896 & 184.589 & 3817 \\
100 & 30 & 0.931 & 0.962 & 183.717 & 3495 \\
100 & 50 & 0.954 & 0.970 & 183.428 & 3198 \\
100 & 100 & 0.968 & 0.976 & 180.592 & 2640 \\
100 & 200 & 0.975 & 0.979 & 177.441 & 2154 \\
200 & 10 & 0.771 & 0.919 & 195.511 & 4556 \\
200 & 30 & 0.958 & 0.971 & 195.709 & 4393 \\
200 & 50 & 0.973 & 0.980 & 193.661 & 3946 \\
200 & 100 & 0.982 & 0.985 & 189.554 & 3244 \\
200 & 200 & 0.986 & 0.988 & 188.616 & 2991 \\
\hline & & & & & \\
& & & & &
\end{tabular}

Table 6: Average correlation coefficients between $\left\{F_{t}\right\}_{t=1}^{T}$ and $\left\{\hat{F}_{\text {Song }, t}\right\}_{t=1}^{T}$ and $\left\{\hat{F}_{A M G, t}\right\}_{t=1}^{T}$ in the case of heterogeneous slope. Iter and Fail indicate the average number of iterations and the number of failures (lack of convergence) of the iterative process for the estimation method of Song (2013), respectively. 


\begin{tabular}{|c|c|c|c|c|c|c|c|c|c|}
\hline \multirow[b]{2}{*}{$n$} & \multirow[b]{2}{*}{$T$} & \multicolumn{2}{|c|}{ Inf MG } & \multicolumn{2}{|c|}{ CCEMG } & \multicolumn{2}{|c|}{ AMG } & \multicolumn{2}{|c|}{ Song } \\
\hline & & $\beta_{1}$ & $\beta_{2}$ & $\beta_{1}$ & $\beta_{2}$ & $\beta_{1}$ & $\beta_{2}$ & $\beta_{1}$ & $\beta_{2}$ \\
\hline 10 & 10 & 0.0285 & 0.0871 & 0.0336 & 0.0864 & 0.0313 & 0.0901 & 0.0309 & 0.0892 \\
\hline 10 & 30 & -0.0104 & 0.0009 & -0.0082 & 0.0019 & -0.0083 & 0.0003 & -0.0088 & 0.0019 \\
\hline 10 & 50 & -0.0299 & 0.0066 & -0.0307 & 0.0066 & -0.0311 & 0.0067 & -0.0314 & 0.0068 \\
\hline 10 & 100 & -0.0153 & -0.0186 & -0.0172 & -0.0185 & -0.0161 & -0.0192 & -0.0175 & -0.0178 \\
\hline 10 & 200 & -0.0274 & -0.0132 & -0.0254 & -0.0134 & -0.0267 & -0.0134 & -0.0245 & -0.0135 \\
\hline 30 & 10 & 0.0001 & -0.0200 & -0.0016 & -0.0235 & -0.0015 & -0.0204 & -0.0004 & -0.0206 \\
\hline 30 & 30 & 0.0186 & -0.0199 & 0.0191 & -0.0194 & 0.0190 & -0.0197 & 0.0197 & -0.0199 \\
\hline 30 & 50 & -0.0350 & 0.0174 & -0.0353 & 0.0175 & -0.0352 & 0.0177 & -0.0354 & 0.0180 \\
\hline 30 & 100 & -0.0130 & -0.0109 & -0.0131 & -0.0102 & -0.0128 & -0.0111 & -0.0132 & -0.0099 \\
\hline 30 & 200 & -0.0472 & -0.0107 & -0.0465 & -0.0105 & -0.0474 & -0.0107 & -0.0463 & -0.0107 \\
\hline 50 & 10 & 0.0296 & -0.0404 & 0.0315 & -0.0383 & 0.0318 & -0.0407 & 0.0304 & -0.0398 \\
\hline 50 & 30 & -0.0335 & 0.0002 & -0.0328 & -0.0008 & -0.0330 & -0.0001 & -0.0326 & -0.0007 \\
\hline 50 & 50 & -0.0070 & 0.0288 & -0.0065 & 0.0288 & -0.0068 & 0.0289 & -0.0061 & 0.0291 \\
\hline 50 & 100 & -0.0475 & 0.0016 & -0.0467 & 0.0021 & -0.0476 & 0.0018 & -0.0468 & 0.0023 \\
\hline 50 & 200 & -0.0057 & -0.0136 & -0.0056 & -0.0138 & -0.0059 & -0.0136 & -0.0051 & -0.0140 \\
\hline 100 & 10 & -0.0155 & -0.0312 & -0.0164 & -0.0295 & -0.0160 & -0.0309 & -0.0145 & -0.0295 \\
\hline 100 & 30 & -0.0003 & -0.0219 & -0.0007 & -0.0221 & -0.0004 & -0.0217 & -0.0002 & -0.0217 \\
\hline 100 & 50 & 0.0006 & -0.0039 & 0.0004 & -0.0043 & 0.0004 & -0.0042 & 0.0003 & -0.0046 \\
\hline 100 & 100 & -0.0146 & -0.0024 & -0.0151 & -0.0022 & -0.0146 & -0.0024 & -0.0149 & -0.0019 \\
\hline 100 & 200 & -0.0234 & -0.0093 & -0.0236 & -0.0097 & -0.0234 & -0.0093 & -0.0237 & -0.0101 \\
\hline 200 & 10 & 0.0171 & -0.0082 & 0.0179 & -0.0092 & 0.0175 & -0.0084 & 0.0182 & -0.0090 \\
\hline 200 & 30 & 0.0061 & 0.0198 & 0.0057 & 0.0200 & 0.0060 & 0.0198 & 0.0062 & 0.0199 \\
\hline 200 & 50 & -0.0073 & -0.0018 & -0.0074 & -0.0018 & -0.0072 & -0.0017 & -0.0074 & -0.0022 \\
\hline 200 & 100 & 0.0238 & -0.0008 & 0.0241 & -0.0005 & 0.0239 & -0.0007 & 0.0241 & -0.0005 \\
\hline 200 & 200 & 0.0249 & 0.0082 & 0.0253 & 0.0082 & 0.0250 & 0.0083 & 0.0256 & 0.0083 \\
\hline
\end{tabular}

Table 7: Bias of $\beta_{i}$ estimators in the case of heterogeneous slope with unobserved common factors only. The DGP is in (20) and (21). Inf $M G$ is the mean group estimators obtained with unobserved common factors. CCEMG is the mean group CCE estimator by Pesaran (2006). AMG is the mean group estimator (8). Song is the Interactive Fixed Effects estimator by Bai (2009) extended to the heterogenous case by Song (2013). 


\begin{tabular}{rrcccccccc}
\hline & \multicolumn{4}{c}{ Inf MG } & \multicolumn{2}{c}{ CCEMG } & \multicolumn{2}{c}{ AMG } & \multicolumn{2}{c}{ Song } \\
\hline$n$ & $T$ & $\beta_{1}$ & $\beta_{2}$ & $\beta_{1}$ & $\beta_{2}$ & $\beta_{1}$ & $\beta_{2}$ & $\beta_{1}$ & $\beta_{2}$ \\
\hline 10 & 10 & 0.2797 & 0.2861 & 0.3646 & 0.3596 & 0.3014 & 0.2985 & 0.2562 & 0.2550 \\
10 & 30 & 0.1283 & 0.1265 & 0.1484 & 0.1456 & 0.1407 & 0.1378 & 0.1541 & 0.1493 \\
10 & 50 & 0.0934 & 0.0930 & 0.1124 & 0.1153 & 0.1048 & 0.1056 & 0.1284 & 0.1306 \\
10 & 100 & 0.0669 & 0.0648 & 0.0900 & 0.0897 & 0.0752 & 0.0745 & 0.1135 & 0.1135 \\
10 & 200 & 0.0447 & 0.0460 & 0.0796 & 0.0794 & 0.0544 & 0.0546 & 0.1071 & 0.1063 \\
30 & 10 & 0.1630 & 0.1624 & 0.1998 & 0.2005 & 0.1644 & 0.1653 & 0.1462 & 0.1437 \\
30 & 30 & 0.0728 & 0.0732 & 0.0809 & 0.0818 & 0.0756 & 0.0763 & 0.0855 & 0.0872 \\
30 & 50 & 0.0543 & 0.0543 & 0.0639 & 0.0632 & 0.0570 & 0.0564 & 0.0743 & 0.0740 \\
30 & 100 & 0.0378 & 0.0375 & 0.0528 & 0.0511 & 0.0400 & 0.0395 & 0.0677 & 0.0659 \\
30 & 200 & 0.0263 & 0.0262 & 0.0441 & 0.0436 & 0.0281 & 0.0278 & 0.0608 & 0.0603 \\
50 & 10 & 0.1262 & 0.1258 & 0.1574 & 0.1560 & 0.1285 & 0.1277 & 0.1137 & 0.1132 \\
50 & 30 & 0.0555 & 0.0565 & 0.0623 & 0.0613 & 0.0570 & 0.0579 & 0.0667 & 0.0661 \\
50 & 50 & 0.0424 & 0.0421 & 0.0494 & 0.0497 & 0.0439 & 0.0435 & 0.0580 & 0.0582 \\
50 & 100 & 0.0291 & 0.0295 & 0.0400 & 0.0392 & 0.0301 & 0.0301 & 0.0513 & 0.0506 \\
50 & 200 & 0.0201 & 0.0198 & 0.0341 & 0.0341 & 0.0210 & 0.0206 & 0.0478 & 0.0476 \\
100 & 10 & 0.0892 & 0.0906 & 0.1095 & 0.1111 & 0.0896 & 0.0916 & 0.0812 & 0.0804 \\
100 & 30 & 0.0401 & 0.0401 & 0.0433 & 0.0436 & 0.0407 & 0.0405 & 0.0467 & 0.0466 \\
100 & 50 & 0.0295 & 0.0294 & 0.0347 & 0.0348 & 0.0299 & 0.0300 & 0.0410 & 0.0409 \\
100 & 100 & 0.0206 & 0.0206 & 0.0274 & 0.0283 & 0.0209 & 0.0209 & 0.0353 & 0.0365 \\
100 & 200 & 0.0144 & 0.0143 & 0.0244 & 0.0239 & 0.0146 & 0.0146 & 0.0338 & 0.0335 \\
200 & 10 & 0.0639 & 0.0636 & 0.0769 & 0.0778 & 0.0640 & 0.0631 & 0.0570 & 0.0570 \\
200 & 30 & 0.0283 & 0.0284 & 0.0315 & 0.0305 & 0.0285 & 0.0286 & 0.0337 & 0.0328 \\
200 & 50 & 0.0208 & 0.0206 & 0.0244 & 0.0242 & 0.0210 & 0.0208 & 0.0288 & 0.0285 \\
200 & 100 & 0.0147 & 0.0147 & 0.0196 & 0.0199 & 0.0148 & 0.0148 & 0.0257 & 0.0258 \\
200 & 200 & 0.0100 & 0.0101 & 0.0163 & 0.0170 & 0.0101 & 0.0102 & 0.0232 & 0.0242 \\
\hline
\end{tabular}

Table 8: RMSE of $\beta_{i}$ estimators in the case of heterogeneous slope with unobserved common factors only. The DGP is in (20) and (21). Inf $M G$ is the mean group estimators obtained with unobserved common factors. CCEMG is the mean group CCE estimator by Pesaran (2006). AMG is the mean group estimator (8). Song is the Interactive Fixed Effects estimator by Bai (2009) extended to the heterogenous case by Song (2013). 


\begin{tabular}{rr|cccc}
\hline$n$ & $T$ & $\operatorname{Corr}\left(F_{t}, \hat{F}_{\text {Song }, t}\right)$ & $\operatorname{Corr}\left(F_{t}, \hat{F}_{A M G, t}\right)$ & iter & fail \\
\hline 10 & 10 & 0.520 & 0.718 & 200 & 5000 \\
10 & 30 & 0.627 & 0.773 & 200 & 5000 \\
10 & 50 & 0.675 & 0.792 & 200 & 5000 \\
10 & 100 & 0.743 & 0.810 & 200 & 5000 \\
10 & 200 & 0.783 & 0.817 & 200 & 5000 \\
30 & 10 & 0.631 & 0.874 & 200 & 5000 \\
30 & 30 & 0.856 & 0.924 & 200 & 5000 \\
30 & 50 & 0.898 & 0.930 & 200 & 5000 \\
30 & 100 & 0.922 & 0.935 & 200 & 5000 \\
30 & 200 & 0.929 & 0.936 & 200 & 5000 \\
50 & 10 & 0.698 & 0.921 & 200 & 5000 \\
50 & 30 & 0.924 & 0.955 & 200 & 5000 \\
50 & 50 & 0.944 & 0.958 & 200 & 5000 \\
50 & 100 & 0.954 & 0.961 & 200 & 5000 \\
50 & 200 & 0.957 & 0.961 & 200 & 5000 \\
100 & 10 & 0.815 & 0.959 & 200 & 5000 \\
100 & 30 & 0.965 & 0.977 & 200 & 5000 \\
100 & 50 & 0.973 & 0.979 & 200 & 5000 \\
100 & 100 & 0.977 & 0.980 & 200 & 5000 \\
100 & 200 & 0.979 & 0.981 & 200 & 5000 \\
200 & 10 & 0.916 & 0.981 & 200 & 5000 \\
200 & 30 & 0.983 & 0.989 & 200 & 5000 \\
200 & 50 & 0.986 & 0.990 & 200 & 5000 \\
200 & 100 & 0.988 & 0.990 & 200 & 5000 \\
200 & 200 & 0.989 & 0.990 & 200 & 5000 \\
\hline & & & & & \\
& & & & &
\end{tabular}

Table 9: Average correlation coefficients between $\left\{F_{t}\right\}_{t=1}^{T}$ and $\left\{\hat{F}_{S o n g, t}\right\}_{t=1}^{T}$ and $\left\{\hat{F}_{A M G, t}\right\}_{t=1}^{T}$ in the case of heterogeneous slope. Iter and Fail indicate the average number of iterations and the number of failures (lack of convergence) of the iterative process for the estimation method of Song (2013), respectively. 


\begin{tabular}{rr|rrrr}
\hline$n$ & $T$ & $\hat{r}_{A M G}$ & $\hat{r}_{\text {Song }}$ & \multicolumn{1}{c}{ iter } & fail \\
\hline 10 & 10 & 3.62 & 2.35 & 171.622 & 3522 \\
10 & 30 & 3.55 & 1.57 & 108.628 & 1236 \\
10 & 50 & 3.60 & 1.52 & 96.416 & 810 \\
10 & 100 & 3.60 & 1.46 & 80.605 & 403 \\
10 & 200 & 3.51 & 1.43 & 72.032 & 222 \\
30 & 10 & 3.58 & 1.64 & 170.493 & 3067 \\
30 & 30 & 2.00 & 1.08 & 94.768 & 369 \\
30 & 50 & 2.00 & 1.39 & 86.301 & 159 \\
30 & 100 & 2.00 & 1.75 & 79.513 & 41 \\
30 & 200 & 2.00 & 1.90 & 74.569 & 0 \\
50 & 10 & 3.38 & 1.29 & 176.557 & 3160 \\
50 & 30 & 2.00 & 1.34 & 108.307 & 361 \\
50 & 50 & 2.00 & 1.64 & 95.829 & 126 \\
50 & 100 & 2.00 & 1.97 & 84.830 & 8 \\
50 & 200 & 2.00 & 2.00 & 79.043 & 10 \\
100 & 10 & 2.74 & 1.04 & 186.556 & 3661 \\
100 & 30 & 2.00 & 1.83 & 127.252 & 374 \\
100 & 50 & 2.00 & 2.00 & 105.139 & 41 \\
100 & 100 & 2.00 & 2.00 & 90.936 & 2 \\
100 & 200 & 2.00 & 2.00 & 85.241 & 0 \\
200 & 10 & 2.06 & 1.00 & 195.032 & 4338 \\
200 & 30 & 2.00 & 2.00 & 137.876 & 337 \\
200 & 50 & 2.00 & 2.00 & 113.399 & 32 \\
200 & 100 & 2.00 & 2.00 & 98.117 & 2 \\
200 & 200 & 2.00 & 2.00 & 91.997 & 2 \\
\hline & & & & &
\end{tabular}

Table 10: Average number of factors estimated by AMG and Song in the case of two unknown common factors. Iter and Fail indicate the average number of iterations and the number of failures (lack of convergence) of the iterative process for the estimation method of Song (2013), respectively. 


\begin{tabular}{rrrrrrrrrr}
\hline & \multicolumn{4}{c}{ Inf MG } & \multicolumn{2}{c}{ CCEMG } & \multicolumn{2}{c}{ AMG } & \multicolumn{2}{c}{ Song } \\
\hline$n$ & \multicolumn{1}{c}{$\beta_{1}$} & \multicolumn{1}{c}{$\beta_{2}$} & \multicolumn{1}{c}{$\beta_{1}$} & \multicolumn{1}{c}{$\beta_{2}$} & \multicolumn{1}{c}{$\beta_{1}$} & \multicolumn{1}{c}{$\beta_{2}$} & \multicolumn{1}{c}{$\beta_{1}$} & \multicolumn{1}{c}{$\beta_{2}$} \\
\hline 10 & 10 & 0.008 & 0.032 & 0.014 & 0.033 & 0.013 & 0.033 & 0.012 & 0.032 \\
10 & 30 & 0.031 & -0.070 & 0.029 & -0.072 & 0.029 & -0.071 & 0.030 & -0.072 \\
10 & 50 & 0.123 & 0.075 & 0.125 & 0.074 & 0.123 & 0.074 & 0.124 & 0.075 \\
10 & 100 & -0.024 & -0.013 & -0.023 & -0.011 & -0.024 & -0.012 & -0.025 & -0.012 \\
10 & 200 & -0.063 & -0.003 & -0.063 & -0.002 & -0.063 & -0.002 & -0.063 & -0.003 \\
30 & 10 & -0.032 & -0.014 & -0.031 & -0.015 & -0.031 & -0.012 & -0.031 & -0.014 \\
30 & 30 & 0.047 & 0.059 & 0.047 & 0.058 & 0.047 & 0.059 & 0.048 & 0.058 \\
30 & 50 & -0.026 & -0.044 & -0.025 & -0.044 & -0.026 & -0.044 & -0.025 & -0.044 \\
30 & 100 & -0.018 & -0.029 & -0.019 & -0.030 & -0.018 & -0.029 & -0.017 & -0.029 \\
30 & 200 & -0.001 & 0.046 & -0.002 & 0.045 & -0.002 & 0.046 & -0.002 & 0.046 \\
50 & 10 & 0.053 & -0.005 & 0.055 & -0.005 & 0.054 & -0.007 & 0.054 & -0.006 \\
50 & 30 & -0.027 & 0.010 & -0.026 & 0.010 & -0.027 & 0.010 & -0.027 & 0.010 \\
50 & 50 & -0.013 & 0.022 & -0.013 & 0.023 & -0.013 & 0.022 & -0.013 & 0.022 \\
50 & 100 & -0.008 & 0.012 & -0.008 & 0.012 & -0.008 & 0.012 & -0.008 & 0.012 \\
50 & 200 & 0.007 & -0.062 & 0.007 & -0.061 & 0.007 & -0.062 & 0.007 & -0.062 \\
100 & 10 & -0.012 & -0.002 & -0.011 & -0.002 & -0.012 & -0.003 & -0.012 & -0.003 \\
100 & 30 & 0.001 & 0.016 & 0.001 & 0.015 & 0.001 & 0.015 & 0.002 & 0.015 \\
100 & 50 & 0.023 & 0.026 & 0.022 & 0.026 & 0.023 & 0.026 & 0.023 & 0.026 \\
100 & 100 & 0.022 & -0.022 & 0.022 & -0.022 & 0.022 & -0.022 & 0.022 & -0.022 \\
100 & 200 & 0.043 & 0.013 & 0.043 & 0.013 & 0.043 & 0.013 & 0.043 & 0.013 \\
200 & 10 & 0.002 & 0.009 & 0.003 & 0.008 & 0.003 & 0.009 & 0.003 & 0.009 \\
200 & 30 & -0.009 & 0.006 & -0.009 & 0.006 & -0.009 & 0.006 & -0.009 & 0.006 \\
200 & 50 & -0.015 & -0.006 & -0.014 & -0.006 & -0.015 & -0.006 & -0.015 & -0.006 \\
200 & 100 & -0.006 & 0.018 & -0.006 & 0.018 & -0.006 & 0.018 & -0.006 & 0.018 \\
200 & 200 & 0.005 & 0.005 & -0.013 & 0.017 & -0.013 & 0.017 & -0.013 & 0.017 \\
\hline
\end{tabular}

Table 11: Bias of $\beta_{i}$ estimators in the case of heterogeneous slope when the number of common factors $f_{t}$ is unknown. Inf $M G$ is the mean group estimators obtained with unobserved common factors. CCEMG is the mean group CCE estimator by Pesaran (2006). AMG is the mean group estimator (8). Song is the Interactive Fixed Effects estimator by Bai (2009) extended to the heterogenous case by Song (2013). 


\begin{tabular}{rrcccccccc}
\hline \multicolumn{4}{c}{ Inf MG } & \multicolumn{2}{c}{ CCEMG } & \multicolumn{2}{c}{ AMG } & \multicolumn{2}{c}{ Song } \\
\hline$n$ & $T$ & $\beta_{1}$ & $\beta_{2}$ & $\beta_{1}$ & $\beta_{2}$ & $\beta_{1}$ & $\beta_{2}$ & $\beta_{1}$ & $\beta_{2}$ \\
\hline 10 & 10 & 0.160 & 0.161 & 0.209 & 0.208 & 0.207 & 0.207 & 0.283 & 0.279 \\
10 & 30 & 0.064 & 0.064 & 0.107 & 0.110 & 0.086 & 0.089 & 0.124 & 0.124 \\
10 & 50 & 0.048 & 0.048 & 0.098 & 0.097 & 0.073 & 0.072 & 0.098 & 0.098 \\
10 & 100 & 0.033 & 0.033 & 0.092 & 0.091 & 0.061 & 0.061 & 0.081 & 0.081 \\
10 & 200 & 0.023 & 0.023 & 0.086 & 0.088 & 0.053 & 0.054 & 0.072 & 0.074 \\
30 & 10 & 0.092 & 0.092 & 0.117 & 0.122 & 0.116 & 0.119 & 0.124 & 0.127 \\
30 & 30 & 0.037 & 0.037 & 0.062 & 0.061 & 0.039 & 0.039 & 0.064 & 0.063 \\
30 & 50 & 0.028 & 0.028 & 0.055 & 0.055 & 0.029 & 0.029 & 0.049 & 0.049 \\
30 & 100 & 0.019 & 0.019 & 0.050 & 0.051 & 0.020 & 0.020 & 0.032 & 0.032 \\
30 & 200 & 0.013 & 0.013 & 0.049 & 0.049 & 0.015 & 0.015 & 0.020 & 0.020 \\
50 & 10 & 0.071 & 0.071 & 0.093 & 0.092 & 0.088 & 0.087 & 0.085 & 0.086 \\
50 & 30 & 0.029 & 0.028 & 0.048 & 0.048 & 0.030 & 0.029 & 0.044 & 0.043 \\
50 & 50 & 0.022 & 0.021 & 0.043 & 0.041 & 0.022 & 0.022 & 0.032 & 0.033 \\
50 & 100 & 0.014 & 0.014 & 0.039 & 0.039 & 0.015 & 0.015 & 0.016 & 0.016 \\
50 & 200 & 0.010 & 0.010 & 0.038 & 0.038 & 0.010 & 0.010 & 0.010 & 0.010 \\
100 & 10 & 0.050 & 0.050 & 0.063 & 0.065 & 0.058 & 0.057 & 0.055 & 0.057 \\
100 & 30 & 0.021 & 0.020 & 0.033 & 0.033 & 0.021 & 0.021 & 0.024 & 0.024 \\
100 & 50 & 0.015 & 0.015 & 0.030 & 0.030 & 0.015 & 0.015 & 0.015 & 0.016 \\
100 & 100 & 0.010 & 0.010 & 0.028 & 0.028 & 0.010 & 0.011 & 0.010 & 0.011 \\
100 & 200 & 0.007 & 0.007 & 0.026 & 0.026 & 0.007 & 0.007 & 0.007 & 0.007 \\
200 & 10 & 0.035 & 0.036 & 0.046 & 0.046 & 0.036 & 0.036 & 0.038 & 0.038 \\
200 & 30 & 0.015 & 0.015 & 0.023 & 0.024 & 0.015 & 0.015 & 0.015 & 0.015 \\
200 & 50 & 0.011 & 0.011 & 0.021 & 0.021 & 0.011 & 0.011 & 0.011 & 0.011 \\
200 & 100 & 0.007 & 0.007 & 0.019 & 0.019 & 0.007 & 0.007 & 0.007 & 0.007 \\
200 & 200 & 0.005 & 0.005 & 0.019 & 0.019 & 0.005 & 0.005 & 0.005 & 0.005 \\
\hline
\end{tabular}

Table 12: RMSE of $\beta_{i}$ estimators in the case of heterogeneous slope when the number of common factors $f_{t}$ is unknown. Inf $M G$ is the mean group estimators obtained with unobserved common factors. CCEMG is the mean group CCE estimator by Pesaran (2006). AMG is the mean group estimator (8). Song is the Interactive Fixed Effects estimator by Bai (2009) extended to the heterogenous case by Song (2013). 


\section{References}

BAI, J. (2003): "Inferential Theory for Factor Models of Large Dimensions," Econometrica, 71(1), $135-171$.

(2009): "Panel Data Models with Interactive Fixed Effects," Econometrica, 77(4), 12291279.

BAI, J., AND S. NG (2002): "Determining the Number of Factors in Approximate Factor Models," Econometrica, 70(1), 191-221.

Bernanke, B., J. Boivin, and P. Eliasz (2005): "Measuring the Effects of Monetary Policy: A Factor-Augmented Vector Autoregressive (FAVAR) Approach," Quarterly Journal of Economics, $120(1), 387-422$.

Castagnetti, C., E. Rossi, and L. Trapani (2012): "Inference on Factor Structures in Heterogeneous Panels," quaderno di dipartimento, n.211, Università di Pavia, Dipartimento di Economia Politica e Metodi Quantitativi.

Chudik, A., M. Pesaran, and E. Tosetti (2011): "Weak and strong cross-section dependence and estimation of large panels," Econometrics Journal, 14(1), 45-90.

Coakley, J., A. Fuertes, and R. Smith (2002): "A Principal Component Approach to CrossSection Dependence in Panels," Discussion paper, Birkbeck College.

Kapetanios, G., and M. Pesaran (2007): “Alternative Approaches to Estimation and Inference in Large Multifactor Panels: Small Sample Results with an Application to Modelling of Asset Returns," in The Refinement of Econometric Estimation and Test Procedures: Finite Sample and Asymptotic Analysis, ed. by G. Phillips, and E. Tzavalis. Cambridge University Press, Cambridge.

Kapetanios, G., M. Pesaran, and T. Yamagata (2011): "Panels with nonstationary multifactor error structures," Journal of Econometrics, 160(2), 326-348.

Lin, Z., And Z. BAi (2010): Probability Inequalities. Science Press and Springer-Verlag.

Ludvigson, S., And S. NG (2007): "The Empirical Risk-Return Relation: A Factor Analysis Approach," Journal of Financial Economics, 83, 171-222.

(2009): "Macro Factors in Bond Risk Premia," The Review of Financial Studies, 22(12), $5027-5067$. 
Pesaran, M. (2006): "Estimation and Inference in Large Heterogeneous Panels with a Multifactor Error Structure," Econometrica, 74(4), 967-1012.

Pesaran, M., and R. Smith (1995): "Estimating Long-Run Relationships from Dynamic Heterogeneous Panels," Journal of Econometrics, 68(1), 79-113.

Pesaran, M., and E. Tosetti (2011): "Large panels with common factors and spatial correlation," Journal of Econometrics, 161(2), 182-202.

RAO, P. B. (2009): "Conditional independence, conditional mixing and conditional association," Annals of the Institute of Statistical Mathematics, 61, 441-460.

Song, M. (2013): "Asymptotic theory for dynamic heterogeneous panels with cross-sectional dependence and its applications," Mimeo.

Stock, J., And M. Watson (2005): "Implication of Dynamic Factor Models for VAR Analysis," working paper 11467. 